\title{
High-dimensional finite elements for elliptic problems with multiple scales
}

\section{Report}

\section{Author(s):}

Hoang, V. H.; Schwab, Christoph

Publication date:

2003-10

Permanent link:

https://doi.org/10.3929/ethz-a-004604740

Rights / license:

In Copyright - Non-Commercial Use Permitted

Originally published in:

SAM Research Report 2003-14 


\title{
High-dimensional finite elements for elliptic problems with multiple scales
}

\author{
V.H. Hoang* and C. Schwab
}

Research Report No. 2003-14

October 2003

Seminar für Angewandte Mathematik

Eidgenössische Technische Hochschule

CH-8092 7ïrich

Switzerland

\footnotetext{
*Seminar for Applied Mathematics, ETH Zurich, CH 8092 Zurich, Switzerland; on leave from Gonville and Caius College and Department of Applied Mathematics and Theoretical Physics, University of Cambridge, England. Work performed in the the network 'HMS 2000' HPRN-CT-2000-00109 and supported by the Swiss BBW under grant BBW 01.0025-1

tWork performed in the network 'Breaking Complexity' HPRN-CT-2002-00286 and supported by the Swiss BBW under grant BBW 02.0418
} 


\title{
High-dimensional finite elements for elliptic problems with multiple scales \\ V.H. Hoang* and C. Schwab \\ Seminar für Angewandte Mathematik \\ Eidgenössische Technische Hochschule \\ CH-80.92 7ïrich \\ Switzerland
}

Research Report No. 2003-14 October 2003

\begin{abstract}
:
Elliptic homogenization problems in a domain $\Omega \subset \mathbb{R}^{d}$ with $n+1$ separated scales are reduced to elliptic one-scale problems in dimension $(n+1) d$. They are discretized by a sparse tensor product finite element method (FEM) which resolves all scales of the solution throughout the physical domain. We prove that this FEM has accuracy, work and memory requirement comparable of FEM for single scale problems in the physical domain $\Omega$ and performs independently of the scale parameters. Numerical examples for problems with two and three scales confirm our results.
\end{abstract}

* Seminar for Applied Mathematics, ETH Zurich, CH 8092 Zurich, Switzerland; on leave from Gonville and Caius College and Department of Applied Mathematics and Theoretical Physics, University of Cambridge, England. Work performed in the the network 'HMS 2000' HPRN-CT-2000-00109 and supported by the Swiss BBW under grant BBW 01.0025-1

'Work performed in the network 'Breaking Complexity' HPRN-CT-2002-00286 and supported by the Swiss BBW under grant BBW 02.0418 
1. Introduction. The numerical solution of problems with multiple scales has attracted increasing attention in recent years. Roughly speaking, we are dealing with problems where significant physical phenomena occur on length scales which differ by several orders of magnitude so that their complete resolution within a single numerical simulation is either impossible or extremely costly.

Most approaches to obtain a computationally tractable model are therefore based on scale separation: the ratio between fine and coarse scale tends to zero asymptotically. For example, classical homogenization uses scale separation for a-priori scale elimination, i.e. the analytical elimination of fine scales from the mathematical model. This involves the derivation of the fine scales' effect on the macroscopic, coarse scales of the solution and the analytic derivation of an 'effective' or 'upscaled' homogenized model equation which governs the coarse solution scales. Numerous mathematical tools to achieve this have been developed, starting with the classical two-scale asymptotic expansion technique $[5,7,6,15,22]$ for linear elliptic problems in divergence form to weak and Gamma convergence techniques for fully nonlinear variational problems with multiple scales (see $[16,11,15]$ and the references there). The homogenized limiting problem on the coarse scale can be solved numerically by standard methods. These numerical solutions do not allow, however, to extract fine scale information on the physical solution. This is only possible if 'corrector' problems, which are again of multiscale type, are solved numerically. The numerical solution of these 'corrector' problems requires full resolution of the fine scales and is as complex as the direct solution of the original problem.

An alternative approach is the direct numerical simulation of the multiscale problem by numerically probing the fine scales only where needed in the coarse scale numerical simulation. If only the coarse solution scales are of interest, this can be done by the so-called Hierarchical Multiscale Methods proposed recently in [12].

If full scale resolution is required, one could incorporate the solution's fine scale behaviour into the trial spaces of a coarse scale FEM, as proposed in [14]. Here, problem adapted shape functions which incorporate the solution's fine scale behaviour must be precomputed. This allows for parallelization, but still has complexity equal to that of a full scale resolution computation and is analyzed only for two-scale problems. Its extension to elliptic problems with multiple scales remains yet to be done.

In the present paper, we propose an alternative approach to the numerical solution of elliptic problems with multiple scales. It is based on the FE discretization of a onescale limit problem in high dimension which was derived first in $\lceil 1,2]$. As above, we require scale separation. Contrary to the above multiscale discretization schemes, however, our method, proposed first in [19], does not require the a-priori determination of the homogenized limiting problem. Like the homogenized limiting problem, the high-dimensional limit problem used here is independent of the scale parameters, and gives the correct homogenized solution. Unlike the homogenized limit problem, the solution of the high-dimensional limit problem provides, to leading order, complete information on the physical solution's oscillations throughout the physical domain. This comes at the price of high dimension of the limiting problem: if the physical problem in $\mathbb{R}^{d}$ has $n+1$ length scales, the limit problem is elliptic and independent of the scale parameters, but posed on a tensorized domain in $\mathbb{R}^{(n+1) d}$. Therefore, it can in principle be solved robustly with a tensor product FEM in $\mathbb{R}^{(n+1) d}$; in practice, however, this is unrealistic due to the high complexity of FEM in dimension $>3$.

Here, we break this high complexity by the use of so-called sparse tensor product $F E$-spaces also known in approximation theory as hyperbolic cross approximations.

Apart from offering a new approach to the numerical solution of elliptic multiscale problems in divergence form, solving a single-scale problem in high dimension numerically is often the only possible approach. For example, in the double porosity problem considered in $[4]$, a simple effective equation in $\mathbb{R}^{3}$ cannot be deduced and the limit problem must be formulated in $\mathbb{R}^{6}$. A first attempt to tackle this high dimensional limit problem numerically was made by T. Arbogast in [3]. There, equations 
depending on $x$ and $y$ in $\mathbb{R}^{3}$ and the time variable $t$ are solved first as equations for $y$ by fixing $x \in \mathbb{R}^{3}$ and then as equations for $x$. The high cost of this approach was handled in [3] by massively parallel solution techniques. It was ultimately limited by the sheer amount of solution data: for each macroscropic degree of freedom, a full microscopic solution must be computed to describe the solution at all scales.

Our approach solves also this data problem: interpreting e.g. a 2-scale solution $u(x, x / \varepsilon)$ as a map from the 'macro'-variable $x \in \Omega$ into the microscopic, or fast, variable $y=x / \varepsilon \in Y$, our sparse tensor product $\mathrm{FE}$ approximation resolves the $x$ and $y$ dependence of $u(x, y)$ throughout $\Omega \times Y$ and acounts for the scale interaction in a very economial way. This strategy is, as we show, only successful if $u(x, y)$ is, as map $\Omega \rightarrow Y$ from the slow into the fast scale, sufficiently smooth (see also [17]). We show here (for scale-separated problems with any finite number of scales) that for the highdimensional one-scale problem this regularity holds which implies numerical solvability in (memory and CPU) complexity comparable to that of fast elliptic one-scale solvers in $\mathbb{R}^{d}$ and independent of the length scale parameters. We consider here only linear, scalar elliptic problems in divergence form and assume full elliptic regularity; the idea of a numerically sparse full scale resolution, however, is applicable to elliptic systems, problems with corners (see [20] for sparse tensor product spaces of FEM with mesh refinement) - this shall be dealt with elsewhere.

Throughout the paper, we employ standard notations: by $H^{k}(\Omega)$ we denote the space of generalized functions on $\Omega$ whose derivatives of orders from 0 to $k$ belong to $L^{2}(\Omega) ; H_{\#}^{k}(Y)$ denotes the subspace of functions in $H_{\mathrm{loc}}^{k}\left(\mathbb{R}^{d}\right)$ that are periodic with respect to the unit cell $Y$, and $C_{\#}^{1}(Y)$ denotes the space of $Y$-periodic $C^{1}$ functions.

The outline of the paper is as follows. In the next Section we state the elliptic multiscale problem and we introduce the high dimensional limit equation, based on notations and results on multiscale convergence from Allaire and Briane [2]. In Section 3 , we define the hierarchic FE spaces in Subsection 3.1 and introduce the tensor product FEM in Subsection 3.2. We prove that sparse tensor product FEM can achieve convergence rates comparable to that of the full tensor product FEM with a number $N$ of degrees of freedom that equals that of a one-scale FEM in $\mathbb{R}^{d}$, under the assumption of a certain multiscale regularity. In Section 4 we prove that this multiscale regularity (which is necessary to obtain optimal convergence rates with the reduced number of degrees of freedom of the sparse FEM) typically holds for the multiscale limit equations, under mild smoothness assumptions on the coefficients of the multiscale elliptic equation and of the domain. In Section 5, we address the implementation of the sparse FEM. Due to the structure of the basis, the stiffness matrix of the sparse FEM is rather densely populated and its explicit formation and storage would require $O\left(N^{2}\right)$ memory. We show how to generate this matrix in a factored form that requires essentially* $O(N)$ memory and that allows to realize a matrix-vector multiplication in essentially $O(N)$ operations. Accordingly, the linear systems for the high dimensional limit problem are solved iteratively, e.g. by cg or GMRES; we present a preconditioner that gives uniformly bounded condition numbers and a solution algorithm for the multidimensional problem of log-linear complexity. In Section 6 we report numerical results confirming our theory.

2. Homogenization problem. In the present section we formulate the elliptic homogenization with multiple scales and state the multiple scale limiting problem as well as an error estimate between the solution of the limiting problem and the solution of the physical problem.

2.1. Problem formulation. Let $\Omega\left\ulcorner\mathbb{R}^{d}\right.$ be a bounded domain and let

$$
A\left(x, y_{1}, \ldots, y_{n}\right) \in L^{\infty}\left(\Omega, C\left(\mathbb{R}^{n d}\right)\right)^{d \times d}
$$

\footnotetext{
*Throughout, "essentially" means up to powers of $\log N$ or of $\log h$ which will be made precise
} in the proofs 
be a matrix function depending on $n+1$ variables and taking values in the space $\mathbb{R}_{s y m}^{d \times d} ; A$ is assumed periodic with respect to $y_{i}$ with the period being the unit cell $Y=[0,1]^{d}$ for each $i=1, \ldots, n$. We assume that $A$ is bounded and uniformly positive definite, i.e there is a constant $\gamma>0$ independent of $\varepsilon$ such that for all vectors $\xi \in \mathbb{R}^{d}$

$$
\gamma|\xi|^{2} \leq \xi^{\mathrm{T}} A \xi \leq \gamma^{-1}|\xi|^{2}
$$

for all $x \in \Omega$ and $y_{i} \in Y, i=1, \ldots, n$.

For a scale parameter $\varepsilon>0$ we consider in $\Omega$ the Dirichlet problem

$$
-\operatorname{div} A^{\varepsilon} \nabla u^{\varepsilon}=f
$$

where $u^{\varepsilon}=0$ on $\partial \Omega$ and $f \in L^{2}(\Omega)$. The $d \times d$ matrix $A^{\varepsilon}$ is assumed to depend on $\varepsilon$ with multiple scales in the following sense: there are $n$ positive functions $\varepsilon_{1}, \ldots, \varepsilon_{n}$ of $\varepsilon$ that converge to 0 when $\varepsilon \rightarrow 0$ and are scale separated in the sense that

$$
\lim _{\varepsilon \rightarrow 0} \varepsilon_{i+1} / \varepsilon_{i}=0
$$

for all $i=1, \ldots, n-1$ and that there is $A\left(x, y_{1}, \ldots, y_{n}\right)$ such that for all $x \in \Omega$

$$
A^{\varepsilon}(x)=A\left(x, \frac{x}{\varepsilon_{1}}, \ldots, \frac{x}{\varepsilon_{n}}\right) .
$$

When $n=1$ we have the classical two scale homogenization problem

$$
-\operatorname{div} A\left(x, \frac{x}{\varepsilon}\right) \nabla u^{\varepsilon}=f
$$

which is dealt with thoroughly in the book by Bensoussan, Lions and Papanicolaou [7]. The purpose of homogenization is to study the limit of $u^{\varepsilon}$ when $\varepsilon$ converges to 0 and to get the information on how $u^{\varepsilon}$ oscillates with respect to $\varepsilon$. The original procedure presented in $[7]$ is to perform a formal multiscale (two scale for the problem (2.4)) asymptotic expansion and then to justify the convergence using the energy method due to L. Tartar [22]. This method has been widely used for many homogenization problems involving two scales but for multiple scales it can only solve a very narrow class of problems and becomes very complicated (see [2] for details). Indeed these two separated processes of performing the asymptotic expansion and using the energy method can be incorporated into one single and quite elegant method of two scale convergence (for two scale problems) originally due to Nguetseng [18] and developed further by Allaire $[1]$ and generalized to multiscale convergence by Allaire and Briane [2]. The multidimensional limiting equation derived in [2] contains, to leading order, complete information on the physical solution's oscillations on all length scales.

We remark that recently Cioranescu et al. [9] introduced the notion of unfolding homogenization that can also be used to deduce our limiting equation and corrector results.

We review next some known results on this limiting equation for which we develop below an efficient. FFM.

2.2. High-dimensional Limit Problem. A key ingredient of homogenization of elliptic problems with multiple scales is the notion of multiscale convergence. We present the definition here, based on Allaire and Briane [2]. To this end, by $Y_{1}, \ldots, Y_{n}$ we denote $n$ unit cells for the $n$ fast scales, and by $C_{\#}\left(Y_{1} \times \ldots \times Y_{n}\right)$ the space of continuous functions by $\phi\left(y_{1}, \ldots, y_{n}\right)$, which are $Y_{k}$ periodic with respect to $y_{k}$ for $k=1, \ldots, n$. For simplicity of notation only, we assume here $Y_{j}=Y=(0,1)^{d}$ : $j=1, \ldots, n$.

Definition 2.1. ([2] Definition 2.3) A sequence $\left\{u^{\varepsilon}\right\}_{\varepsilon} \subset L^{2}(\Omega)(n+1)-$ scale converges to $u_{0}\left(x, y_{1}, \ldots, y_{n}\right) \in L^{2}\left(\Omega \times Y_{1} \times \ldots Y_{n}\right)$ if

$$
\begin{aligned}
\lim _{\varepsilon \rightarrow 0} \int_{\Omega} u^{\varepsilon} \phi\left(x, \frac{x}{\varepsilon_{1}}, \ldots, \frac{x}{\varepsilon_{n}}\right) d x= \\
\quad \int_{\Omega} \int_{Y_{1}} \ldots \int_{Y_{n}} u_{0}\left(x, y_{1}, \ldots, y_{n}\right) \phi\left(x, y_{1}, \ldots, y_{n}\right) d x d y_{1} \ldots d y_{n} .
\end{aligned}
$$


for any function $\phi \in L^{2}\left(\Omega, C_{\#}\left(Y_{1} \times \ldots \times Y_{n}\right)\right)$.

The relevance of this definition lies in the following compactness result.

THEовем 2.2. ([2] Theorem 2.5) Under the assumption (2.3) of scale separation, from each bounded sequence in $L^{2}(\Omega)$ we can extract a subsequence which $(n+1)-$ scale converges, as $\varepsilon \rightarrow 0$, to a function $u_{0} \in L^{2}\left(\Omega \times Y_{1} \times \ldots \times Y_{n}\right)$.

We define the space $\mathbf{V}$ as

$$
\mathbf{V}=\left\{\left(\phi,\left\{\phi_{i}\right\}\right): \phi \in H_{0}^{1}(\Omega), \phi_{i} \in L^{2}\left(\Omega \times Y_{1} \times \ldots \times Y_{i-1}, H_{\#}^{1}\left(Y_{i}\right) / \mathbb{R}\right)\right\}
$$

endowed with the norm

$$
\left\|\left(\phi,\left\{\phi_{i}\right\}\right)\right\|\|=\| \nabla \phi\left\|_{L^{2}(\Omega)}+\sum_{i=1}^{n}\right\| \nabla_{y_{i}} \phi_{i} \|_{L^{2}\left(\Omega \times Y_{1} \times \ldots \times Y_{i}\right)}
$$

for $\left(\phi, \phi_{1}, \ldots, \phi_{i}\right) \in \mathbf{V}$. Then for the problem (2.2) we have the following result.

THEOREM 2.3. The solution $u^{\varepsilon}$ of the problem (2.2) converges weakly to a function $u$ in $H_{0}^{1}(\Omega)$ and the gradient $\nabla u^{\varepsilon}(n+1)$-scale converges to the limit

$$
\nabla u(x)+\sum_{i=1}^{n} \nabla_{y_{i}} u_{k}\left(x, y_{1}, \ldots, y_{i}\right)
$$

where $\left(u, u_{1}, \ldots, u_{n}\right)$ is the unique solution in the space $\mathbf{V}$ of the variational problem

$$
\begin{aligned}
& B\left(u,\left\{u_{i}\right\} ; \phi,\left\{\phi_{i}\right\}\right) \\
& =\int_{\Omega} \int_{Y_{1}} \cdots \int_{Y_{n}} A\left(\nabla_{x} u+\sum_{i=1}^{n} \nabla_{y_{i}} u_{i}\right) \cdot\left(\nabla_{x} \phi+\sum_{i=1}^{n} \nabla_{y_{i}} \phi_{i}\right) d x d y_{1} \ldots d y_{n} \\
(2.7) & =\int_{\Omega} f \phi d x \quad \forall\left(\phi,\left\{\phi_{i}\right\}\right) \in \mathrm{V} .
\end{aligned}
$$

The bilinear form $B$ is continuous and coercive in $\mathrm{V}$ : there are $c_{1}, c_{2}>0$ independent of $\varepsilon$ such that

$$
\forall\left(\phi,\left\{\phi_{i}\right\}\right) \in \mathbf{V}: \quad B\left(\phi,\left\{\phi_{i}\right\} ; \phi,\left\{\phi_{i}\right\}\right) \geq c_{1}\left\|\mid\left(\phi,\left\{\phi_{i}\right\}\right)\right\| \|^{2},
$$

$(2.9) \forall\left(u,\left\{u_{i}\right\}\right),\left(v,\left\{v_{i}\right\}\right) \in \mathbf{V}: \quad B\left(u,\left\{u_{i}\right\} ; v,\left\{v_{i}\right\}\right) \leq c_{2}\left\|\mid\left(u,\left\{u_{i}\right\}\right)\right\|\|\|.\left(v,\left\{v_{i}\right\}\right)\|\|$.

For a proof, we refer to ([2] Theorem 2.11, equation (2.9)).

REMARK 1. The limit problem (2.7) is independent of the scale parameter $\varepsilon$, but high dimensional. We can also deduce a limiting equation for $u(x)$ only and express $u_{i}$ in terms of $u$ and of the solutions of appropriate cell problems. This will be done in Section 4 ahead. For example, for a two scale problem, i.e for $n=1$, equation (2.7) beromes

$$
\int_{\Omega} \int_{Y} A\left(\nabla_{x} u+\nabla_{y} u_{1}\right) \cdot\left(\nabla_{x} \phi+\nabla_{y} \phi_{1}\right) d x d y=\int_{\Omega} f \phi
$$

for all $\phi \in H_{0}^{1}(\Omega)$ and $\phi_{1} \in L^{2}\left(\Omega, H_{\#}^{1}(Y) / \mathbb{R}\right)$. This limit problem is derived in Allaire $\lceil 1\rceil$.

Next we mention a corrector result to illustrate how $u^{\varepsilon}$ can be approximated in the physical domain $\Omega$ in terms of $u_{0}, u_{1}, \ldots, u_{n}$.

Theorem 2.4. ([2/ Theorem 2.14) Assume that the solution $\left(u, u_{1}, \ldots, u_{n}\right)$ of the problem (2.7) is smooth, say $u \in C^{1}(\bar{\Omega})$ and $u_{i} \in C^{1}\left(\bar{\Omega}, C_{\#}^{1}\left(Y_{1} \times \ldots \times Y_{i}\right)\right)$ for all $i=1, \ldots, n$. Then

$$
u^{\varepsilon}(x)-\left[u(x)+\sum_{i=1}^{n} \varepsilon_{i} u_{i}\left(x, \frac{x}{\varepsilon_{1}}, \ldots, \frac{x}{\varepsilon_{i}}\right)\right] \rightarrow 0
$$


strongly in $H^{1}(\Omega)$.

REMARK 2 . If the data $A, \Omega, f$ are smooth then the functions $u, u_{i}$ are all smooth. We can also pass to the limit for some classes of non smooth matrices $A$. This leads to the non smoothness of $u_{k}$ but nevertheless a corrector can always be found using the 'inverse unfolding operator' due to Cioranescu et al. [9]. Details about which matrices $A$ are "admissible" may be found in $[2]$ and $[9]$.

3. FEM discretization. We present FE discretizations of the limit problem (2.7). Since this problem is elliptic and posed in the product domain $\Omega \times Y_{1} \times \ldots \times Y_{n}$. it is natural to discretize (2.7) with tensor product finite elements. We treat the full tensor product and the sparse tensor product space separately to highlight their differences. Error estimates follow then straightforwardly from approximation theory in the component domains $\Omega$ and $Y_{k}$.

3.1. Hierarchic FE spaces. For the construction of the sparse tensor product FE spaces in $\Omega \times Y_{1} \times \ldots \times Y_{n}$, we require hierarchic sequences of $\mathrm{FE}$ spaces in the component domains. We sketch exemplarily two constructions of such subspace sequences. Consider a sequence $\left\{V^{l}\right\}_{l=0}^{\infty}$ of subspaces of $H^{1}(\Omega)$ and a sequence $\left\{V_{\#}^{l}\right\}_{l=0}^{\infty}$ of subspaces of $H_{\#}^{1}(Y)$ such that $V^{l} \subset V^{l+1}$ and $V_{\#}^{l} \subset V_{\#}^{l+1}$ for all $l=0,1, \ldots$ Assuming that $\Omega$ is a bounded Lipschitz polyhedron with plane sides, we divide $\Omega$ into simplices (triangles in dimension $d=2$ and tetrahedra in dimension $d=3$ ). For each $l$ each element of $V^{l}$ restricted to each of these simplices is a polynomial. Similarly $Y$ is divided to simplices; and each element of $V_{\#}^{l}$ restricted to each of these simplices is a polynomial. We consider the following cases:

EXAMPLE 1. (h-FEM) Let $\left\{\mathcal{T}^{l}\right\}_{l=0}^{\infty}$ be a nested sequence of regular simplices of $\Omega: \Omega$ is divided into a regular family $\mathcal{T}^{0}=\{\mathcal{T}\}$ of simplices; then we define $\mathcal{T}^{l}$ recursively by dividing each simplex in $\mathcal{T}^{l-1}$ into 4 congruent triangles for $d=2$ and 8 congruent tedrahedra for $d=3$. The nested sequence $\left\{\mathcal{T}_{\#}^{l}\right\}_{l=0}^{\infty}$ of regular simplices distributed periodically in $Y$ is defined similarly. For $p \geq 1$ a polynomial degree, and $l \geq 0$ a refinement level, we define the finite element spaces

$$
\begin{gathered}
V^{l}=\left\{u \in H^{1}(\Omega):\left.u\right|_{K} \in \mathcal{P}_{p}(K) \forall K \in \mathcal{T}^{l}\right\}, \\
V_{\#}^{l}=\left\{u \in H_{\#}^{1}(Y):\left.u\right|_{K} \in \mathcal{P}_{p}(K) \forall K \in \mathcal{T}_{\#}^{l}\right\}, \\
V_{0}^{l}=\left\{u \in H_{0}^{1}(\Omega):\left.u\right|_{K} \in \mathcal{P}_{p}(K) \forall K \in \mathcal{T}^{l}\right\}
\end{gathered}
$$

where $\mathcal{P}_{p}(K)$ denotes the set of polynomials of degree not exceeding $p$ in $K$.

For these spaces, we recall the approximation properties. Let $h_{l}=O\left(2^{-l}\right)$ denote the meshwidth. Then, for all $s \geq 0$ and $t \geq 0$ we have (e.g. [8])

$$
\begin{gathered}
\inf _{v \in V^{l}}\|u-v\|_{H^{1}(\Omega)} \leq c h_{l}^{\min (s, p)}\|u\|_{H^{s+1}(\Omega)}, \\
\inf _{v \in V^{l}}\|u-v\|_{L^{2}(\Omega)} \leq c h_{l}^{\min (s, p)+1}\|u\|_{H^{s+1}(\Omega)},
\end{gathered}
$$

for all $u \in H^{s+1}(\Omega)$ and

$$
\begin{gathered}
\inf _{v \in V_{\#}^{l}}\|u-v\|_{H^{1}(Y)} \leq c h_{l}^{\min (t, p)}\|u\|_{H^{t+1}(Y)}, \\
\inf _{v \in V_{\#}^{l}}\|u-v\|_{L^{2}(Y)} \leq c h_{l}^{\min (t, p)+1}\|u\|_{H^{t+1}(Y)} .
\end{gathered}
$$

for all $u \in H_{\#}^{t+1}(Y)$. Here, $c=c(t, p)$. 
EXAmple 2. ( $p$-FEM) Let $\Omega$ and $Y$ be divided into simplices $\mathcal{T}$ and a set of periodically distributed simplices $\mathcal{T}_{\#}$, respectively. Let $p=1,2, \ldots$ be the polynomial degree. We define

$$
\begin{gathered}
V^{p}=\left\{u \in H^{1}(\Omega):\left.u\right|_{K} \in \mathcal{P}_{p}(K) \forall K \in \mathcal{T}\right\}, \\
V_{\#}^{p}=\left\{u \in H_{\#}^{1}(Y):\left.u\right|_{K} \in \mathcal{P}_{p}(K) \forall K \in \mathcal{T}_{\#}\right\}, \\
V_{n}^{p}=\left\{u \in H_{0}^{1}(\Omega):\left.u\right|_{K} \in \mathcal{P}_{p}(K) \forall K \in \mathcal{T}\right\} .
\end{gathered}
$$

Then we also have similar approximation properties as in (3.1), (3.2), (3.3) and (3.4) except that instead of the meshwidth $h_{l}$ we have $p$.

3.2. Tensor product FEM. To approximate $u_{i}\left(x, y_{1}, \ldots, y_{i}\right)$ we need FE subspaces of $L^{2}\left(\Omega \times Y_{1} \times \ldots \times Y_{i-1}, H_{\#}^{1}\left(Y_{i}\right)\right)$. Since

$$
L^{2}\left(\Omega \times Y_{1} \times \ldots \times Y_{i-1}, H_{\#}^{1}\left(Y_{i}\right)\right) \cong L^{2}(\Omega) \times L^{2}\left(Y_{1}\right) \times \ldots \times L^{2}\left(Y_{i-1}\right) \times H_{\#}^{1}\left(Y_{i}\right),
$$

a natural choice of a $\mathrm{FE}$ space is the tensor product $\mathrm{FE}$ space

$$
\mathbf{V}^{L}=\left\{\left(u^{L},\left\{u_{i}^{L}\right\}\right): u^{L} \in V_{0}^{L}, u_{i}^{L} \in V_{i}^{L}, i=1, \ldots, n\right\}
$$

where

$$
V_{i}^{L}=V^{L} \otimes \underbrace{V_{\#}^{L} \otimes \ldots \otimes V_{\#}^{L}}_{i \text { times }}
$$

To investigate the convergence rate of the tensor product $\mathrm{FE}$ spaces $V_{i}^{L}$, we quantify the solution's regularity. To describe it, we introduce the space $\mathcal{H}_{i}^{t}$ of functions $w=$ $w\left(x, y_{1}, \ldots, y_{i}\right) \in L^{2}\left(\Omega \times Y_{1} \times \ldots \times Y_{i-1}, H^{1+t}\left(Y_{i}\right)\right)$ that are periodic in $y_{j}$ for $j=$ $1, \ldots, i$ such that $w \in L^{2}\left(Y_{1} \times \ldots \times Y_{i}, H^{t}(\Omega)\right)$ and $w \in L^{2}\left(\Omega \times \Pi_{j \neq k} Y_{j}, H^{t}\left(Y_{k}\right)\right)$ for all $k=1, \ldots, i-1$. We equip $\mathcal{H}_{i}^{t}$ with the norm

$$
\|w\|_{\mathcal{H}_{i}^{t}}=\|w\|_{L^{2}\left(\Omega \times Y_{1} \times \ldots \times Y_{i-1}, H^{1+t}\left(Y_{i}\right)\right)}+\sum_{k=1}^{i-1}\|w\|_{L^{2}\left(\Omega \times \Pi_{j \neq k} Y_{j}, H^{t}\left(Y_{k}\right)\right)} .
$$

Lemma 3.1. If $w \in \mathcal{H}_{i}^{t}$ then

$$
\inf _{v \in V_{i}^{L}}\|w-v\|_{L^{2}\left(\Omega \times Y_{1} \times \ldots \times Y_{i-1}, H^{1}\left(Y_{i}\right)\right)} \leq c h_{l}^{\min (t, p)}\|w\|_{\mathcal{H}_{i}^{t}} .
$$

Proof We first define the following orthogonal projections in the norm of $H^{1}(Y)$, $L^{2}(Y)$ and $L^{2}(\Omega)$ respectively

$$
\begin{gathered}
P_{\#}^{l 1}: H_{\#}^{1}(Y) \longrightarrow V_{\#}^{l}, \\
P_{\#}^{l 0}: L^{2}(Y) \longrightarrow V_{\#}^{l}, \\
P^{l 0}: L^{2}(\Omega) \longrightarrow V^{l} .
\end{gathered}
$$

For simplicity of notation, in the following inequalities we denote by $L_{i}^{2}$ the space $L^{2}\left(\Omega \times Y_{1} \times \ldots \times Y_{i-1}, H^{1}\left(Y_{i}\right)\right)$. We then have

$$
\begin{aligned}
\inf _{v \in V_{i}^{L}}\|w-v\|_{L_{i}^{2}} & \leq\left\|w-i d \otimes P_{\#}^{l 1} w\right\|_{L_{i}^{2}}+\left\|i d \otimes P_{\#}^{l 1} w-i d \otimes P_{\#}^{l 0} \otimes P_{\#}^{l 1} w\right\|_{L_{i}^{2}}+ \\
\ldots & +\left\|i d \otimes P_{\#}^{l 0} \otimes \ldots \otimes P_{\#}^{l 0} \otimes P_{\#}^{l 1} w-P^{l 0} \otimes P_{\#}^{l 0} \otimes \ldots \otimes P_{\#}^{l 1} \omega\right\|_{L_{i}^{2}} \\
& \leq c\left(h_{l}+h_{l}^{\min (s, p)}\right)\|w\|_{\mathcal{H}_{i}^{t}}
\end{aligned}
$$


due to the approximation properties (3.1) - (3.4) and to the boundedness of the projection operators.

The finite element approximation of $\left(u,\left\{u_{i}\right\}\right)$ reads: find $\left(u^{L},\left\{u_{i}^{L}\right\}\right) \in \mathbf{V}^{L}$ such that

$$
B\left(u^{L},\left\{u_{i}^{L}\right\} ; \phi^{L},\left\{\phi_{i}^{L}\right\}\right)=\int_{\Omega} f \phi^{L} d x \quad \forall\left(\phi^{L},\left\{\phi_{i}^{L}\right\}\right) \in \mathbf{V}^{L} .
$$

By (2.8), (2.9), it satisfies the quasi optimal error estimate

$$
\left\|\left(u-u^{L},\left\{u_{i}-u_{i}^{L}\right\}\right)\right\| \mid \leq \inf _{\left(v^{L},\left\{v_{i}^{L}\right\}\right) \in \mathbf{V}^{L}}\left\|\left(u-v^{L},\left\{u_{i}-v_{i}^{L}\right\}\right)\right\| .
$$

We have immediately from Lemma 3.1

Proposition 3.2. If $u \in H^{2}(\Omega)$ and $u_{i} \in \mathcal{H}_{i}^{1}$ for $i=1, . ., n$, then with the full tensor product finite element spaces in Example 1

$$
\left\|\left(u-u^{L},\left\{u_{i}-u_{i}^{L}\right\}\right)\right\| \mid \leq h_{L}\left(\|u\|_{H^{2}(\Omega)}+\sum_{i=1}^{n}\left\|u_{i}\right\|_{\mathcal{H}_{i}^{1}}\right)
$$

REMARK 3 . If the solution has regularity $u \in H^{t+1}(\Omega)$ and $u_{i} \in \mathcal{H}_{i}^{t}$, we get

$$
\|\|\left(u-u^{L},\left\{u_{i}-u_{i}^{L}\right\}\right) \| \mid \leq c h_{L}^{\min (t, p)}\left(\|u\|_{H^{t+1}(\Omega)}+\sum_{i=1}^{n}\left\|u_{i}\right\|_{\mathcal{H}_{i}^{t}}\right)
$$

REMARK 4. For all $p \geq 1$ we have $N_{L}=\operatorname{dimV}^{L}=O\left(h_{I^{-}}^{-(n+1) d}\right)$.

3.3. Sparse tensor product FEM. The full tensor product FE space is natural for the numerical solution of the limiting problem (2.7). However, since its number of degrees of freedom is $O\left(h_{L}^{-(n+1) d}\right)$, its use is prohibitive in practice, especially in dimension $d=3$ and for more than two scales. In this section we develop the sparse FEM, and prove that its convergence rate is essentially the same as that of the full tensor product FEM but its number of degrees of freedom differs from that of a one scale problem in $\mathbb{R}^{d}$ only by a logarithmic factor of $h_{L}$.

To do so, we define the following increment, or "detail" spaces

$$
W^{l}=\left(P^{l 0}-P^{(l-1) 0}\right) V^{l}, W_{\#}^{l 0}=\left(P_{\#}^{l 0}-P_{\#}^{(l-1) 0}\right) V_{\#}^{l}, W_{\#}^{l 1}=\left(P^{l 1}-P^{(l-1) 1}\right) V_{\#}^{l} ;
$$

Then

$$
V^{l}=\bigoplus_{0 \leq i \leq l} W^{i}, \quad V_{\#}^{l}=\bigoplus_{0 \leq i \leq l} W_{\#}^{i 0}=\bigoplus_{0 \leq i \leq l} W_{\#}^{i 1} .
$$

The full tensor product space $V_{i}^{L}$ defined in (3.6) can then be written as

$$
V_{i}^{L}=\bigoplus_{\substack{0 \leq j_{j} \leq L \\ k=0,1 \ldots, i}} W^{j_{0}} \otimes W_{\#}^{j_{1} 0} \otimes \ldots \otimes W_{\#}^{j_{i-1} 0} \otimes W_{\#}^{j 1} .
$$

and the corresponding sparse tensor product FE spaces are defined by

$$
\hat{V}_{i}^{L}=\bigoplus_{0 \leq j_{0}+\ldots+j_{i} \leq L} W^{j_{0}} \otimes W_{\#}^{j_{1} 0} \otimes \ldots \otimes W_{\#}^{j_{i-1} 0} \otimes W_{\#}^{j 1} .
$$

The sparse FE space for the approximation of (2.7) is then given by

$$
\hat{\mathbf{V}}^{L}=\left\{\left(\hat{u}^{L},\left\{\hat{u}_{i}^{L}\right\}\right): \hat{u}^{L} \in V_{0}^{L}, \hat{u}_{i}^{L} \in \hat{V}_{i}^{L}\right\}
$$


and we approximate the solution $\left(u,\left\{u_{i}\right\}\right)$ of the problem (2.7) by: find $\left(\hat{u}^{L},\left\{\hat{u}_{i}^{L}\right\}\right) \in \hat{\mathbf{V}}^{L}$ such that

$$
B\left(\hat{u}^{L},\left\{\hat{u}_{i}^{L}\right\} ; \hat{\phi}^{L},\left\{\hat{\phi}_{i}^{L}\right\}\right)=\int_{\Omega} f \hat{\phi}_{i}^{L} \quad \forall\left(\hat{\phi}^{L},\left\{\hat{\phi}_{i}^{L}\right\}\right) \in \hat{\mathbf{V}}^{L} .
$$

By $(2.9)$

$$
\left\|\left(u-\hat{u}^{L},\left\{u_{i}-\hat{u}_{i}^{L}\right\}\right)\right\| \mid \leq c \inf _{\left(\hat{\phi}^{L},\left\{\hat{\phi}_{i}^{L}\right\}\right) \in \hat{\mathbf{V}}^{L}}\left\|\left(u-\hat{\phi}^{L},\left\{u_{i}-\hat{\phi}_{i}^{L}\right\}\right)\right\| \|,
$$

where $c$ is independent of $L$.

To derive an error estimate for this space in terms of $h_{L}$, we need suitable approximation results. To state them, we denote for a positive integer $t$ by $\hat{\mathcal{H}}_{i}^{t}$ the space of functions $w\left(x, y_{1}, \ldots, y_{i}\right)$ that are $Y$-periodic in $y_{i}$ for $j=1, \ldots, i$ and that are such that for all $\alpha=\left(\alpha_{0}, \ldots, \alpha_{i}\right)$ with $0 \leq \alpha_{j} \leq t$ for $j=0, \ldots, i-1$ and $0 \leq \alpha_{i} \leq t+1$ holds $\partial^{|\alpha|} /\left(\partial^{\alpha_{0}} x \partial^{\alpha_{1}} y_{1} \ldots \partial^{\alpha_{i}} y_{i}\right) \in L^{2}\left(\Omega \times Y_{1} \times \ldots \times Y_{i}\right)$. We equip $\hat{\mathcal{H}}_{i}^{t}$ with the norm

$$
\|w\|_{\hat{\mathcal{H}}_{i}^{t}}=\sum_{\substack{0 \leq \alpha_{i} \leq t+1 \\ 0 \leq \alpha_{i} \leq t \\(0 \leq j \leq i-1)}}\left\|\frac{\partial^{|\alpha|}}{\partial^{\alpha_{0}} x \partial^{\alpha_{1}} y_{1} \ldots \partial^{\alpha_{i}} y_{i}} w\right\|_{L^{2}\left(\Omega \times Y_{1} \times \ldots \times Y_{i}\right)} .
$$

The definition for non integer $t$ is as usual by interpolation.

LEMMA 3.3. With respect to the finite element spaces defined in Example 1, if $w \in \hat{\mathcal{H}}_{i}^{t}$ then

$$
\inf _{v \in \hat{V}_{i}^{L}}\|w-v\|_{L_{i}^{2}} \leq c(L+1)^{i / 2} h_{L}^{\min (t, p)}\|w\|_{\hat{\mathcal{H}}_{i}^{t}}
$$

where we denote, as in Lemma 3.1, by $L_{i}^{2}$ the space $L^{2}\left(\Omega \times Y_{1} \times \ldots \times Y_{i-1}, H^{1}\left(Y_{i}\right)\right)$. Proof We define the sparse interpolant $\hat{w}^{L}$ of $w$ by

$\hat{w}^{L}=\sum_{0 \leq j_{0}+\ldots+j_{i} \leq L}\left(P^{j_{0}}-P^{j_{0}-1}\right) \otimes\left(P_{\#}^{j_{1} 0}-P_{\#}^{\left(j_{1}-1\right) 0}\right) \otimes \ldots \otimes\left(P_{\#}^{j_{i-1} 0}-P_{\#}^{\left(j_{i-1}-1\right) 0} \otimes\left(P_{\#}^{j_{i} 1}-P_{\#}^{\left(j_{i}-1\right) 1}\right) w ;\right.$ where $P^{-1}=P_{\#}^{(-1) 0}=P_{\#}^{(-1) 1}=0$. Then we have

$$
\begin{aligned}
& \left\|w-\hat{w}^{L}\right\|_{L_{i}^{2}}^{2}=\left\|\sum_{j_{0}+\ldots+j_{i}>L+1}\left(P^{j_{0}}-P^{j_{0}-1}\right) \otimes \ldots \otimes\left(P_{\#}^{j_{i} 1}-P_{\#}^{\left(j_{i}-1\right) 1}\right) w\right\|_{L_{i}^{2}}^{2} \\
& \leq \sum_{j_{0}+\ldots+j_{i} \geq L+1}\left\|\left(P^{j_{0}}-P^{j_{0}-1}\right) \otimes \ldots \otimes\left(P_{\#}^{j_{i} 1}-P_{\#}^{\left(j_{i}-1\right) 1}\right) w\right\|_{L_{i}^{2}}^{2} \\
& \leq c \sum_{j_{0}+\ldots+j_{i} \geq L+1}\left(h_{j_{0}-1} \ldots h_{j_{i}-1}\right)^{2 \min (t, p)}\|w\|_{\hat{\mathcal{H}}_{i}^{t}}^{2} \\
& \leq c 4^{i \min (t, p)} \sum_{j_{0}+\ldots+j_{i}>L+1} 2^{-2\left(j_{0}+\ldots+j_{i}\right) \min (t, p)}\|w\|_{\hat{\mathcal{H}}_{i}^{t}}^{2} \\
& \leq c(L+1)^{i} 2^{-2 L \min (t, p)}\|w\|_{\hat{\mathcal{H}}_{i}^{t}}^{2} .
\end{aligned}
$$

Note that $h_{l}=c 2^{-l}$; we set $h_{-1}=1$. The last inequality is proved as follows:

$$
\begin{aligned}
& \sum_{j_{0}+\ldots+j_{i} \geq L+1} 2^{-2\left(j_{0}+\ldots+j_{i}\right) \min (t, p)}=\sum_{j_{0}, \ldots, j_{i-1} \geq 0} 2^{-2\left(j_{0}+\ldots+j_{i-1}\right) \min (t, p)} \sum_{\substack{j_{i} \geq \max \left(L+1-\\
\left(j_{0}+\ldots+j_{i-1}\right), 0\right)}} 2^{-2 j_{i} \min (t, p)} \\
= & \frac{1}{1-2^{-2 \min (t, p)}} \sum_{j_{0}, \ldots, j_{i-1} \geq 0} 2^{-2 \max \left((L+1), j_{0}+\ldots+j_{i-1}\right)+1} \\
= & \frac{1}{1-2^{-2 \min (t, p)}} \sum_{j_{0}+\ldots+j_{i-1}<L+1} 2^{-2(L+1) \min (t, p)}+
\end{aligned}
$$




$$
\begin{aligned}
& \frac{1}{1-2^{-2 \min (t, p)}} \sum_{j_{0}+\ldots+j_{i-1}>L+1} 2^{-2\left(j_{0}+\ldots+j_{i-1}\right) \min (t, p)} \\
& \leq \frac{(L+1)^{i} 2^{-2(L+1) \min (t, p)}}{1-2^{-2 \min (t, p)}}+\frac{1}{1-2^{-2 \min (t, p)}} \sum_{j_{0}+\ldots+j_{i-1}>L+1} 2^{-2\left(j_{0}+\ldots+j_{i-1}\right) \min (t, p)} \\
& \leq \frac{(L+1)^{i} 2^{-2(L+1) \min (t, p)}}{1-2^{-2 \min (t, p)}}+\frac{(L+1)^{i-1} 2^{-2(L+1) \min (t, p)}}{\left(1-2^{-2 \min (t, p)}\right)^{2}}+ \\
& \quad \frac{1}{\left(1-2^{-2 \min (t, p)}\right)^{2}} \sum_{j 0} 2^{-2\left(j_{0}+\ldots+j_{i-2}\right) \min (t, p)} \\
& \ldots \\
& \leq \operatorname{ci}(L+1)^{i 2^{-2(L+1)} .}
\end{aligned}
$$

where the constant $c$ may depend on $t$ but does not depend on $L$.

From this we have the following estimate for the error.

PROPOSITION 3.4. With respect to the sparse tensor product finite element spaces defined in Example 1, if the solution $\left(u,\left\{u_{i}\right\}\right)$ of the problem (2.7) is such that $u \in$ $H^{2}(\Omega)$ and $u_{i} \in \hat{\mathcal{H}}_{i}^{1}$ for $i=1, \ldots, n$ then

$$
\left\|\left|\left(u-\hat{u}^{L},\left\{u_{i}-\hat{u}_{i}^{L}\right\}\right) \|\right| \leq c(L+1)^{n / 2} h_{L}\left(\|u\|_{H^{2}(\Omega)}+\sum_{i=1}^{n}\left\|u_{i}\right\|_{\hat{\mathcal{H}}_{i}^{1}}\right) .\right.
$$

REMARK 5. If $u,\left\{u_{i}\right\}$ possess higher regularity i.e $u \in H^{1+t}(\Omega)$ and $u_{i} \in \hat{\mathcal{H}}_{i}^{t}$ then we will have $h_{I}^{\min (t, p)}$ in the right hand side. The number of degrees of freedom of the sparse FE space $\hat{\mathbf{V}}^{L}$ is then $O\left(h_{I^{\prime}}^{-d}\left|\log h_{L}\right|^{n}\right)$ which is, up to logarithmic terms, that of the one scale problem i.e when $n=0$.

3.4. Convergence in physical variables. We now estimate the error between the solution $u^{\varepsilon}$ of the physical problem (2.2) in terms of the FE approximations of problem (2.7). We based this on an explicit error estimate between $u^{\varepsilon}$ and the correctors. For the two scale case (i.e. $n=1$ ) we recall that

Proposition 3.5. Assume that $A(x, y) \in C^{\infty}\left(\bar{\Omega}, C_{\#}^{\infty}(Y)\right)_{s y m}^{d \times d}$ and that $\Omega$ is a convex polyhedron. Then

$$
\left\|u^{\varepsilon}-\left(u(x)+\varepsilon u_{1}\left(x, \frac{x}{\varepsilon}\right)\right)\right\|_{H^{1}(\Omega)} \leq C \varepsilon^{1 / 2} .
$$

The constant $C$ is independent of $\varepsilon$ but depends on $u$ and $u_{1}$.

REMARK 6 . This two-scale result is well known. Most of the existing literature $([7,15])$ presents error estimates for the case where $A$ depends only on $y$. Section 1.4 of $[15]$ presents a concise proof under the assumption that the coefficients are smooth. the homogenized solution $u$ is in $C^{2}(\bar{\Omega})$ and the corrector functions $w_{k}$ (see the next section) are in $W^{1, \infty}(Y)$. The error estimate for the case where $A$ also depends on $x$ can be shown simply by following this proof line by line. We need $A$ to depend on $x$ in a smooth manner. A close look at the proof of [15] shows that once the coefficients are assumed to be smooth, it is enough to consider $u \in H^{2}(\Omega)$ as in Chapter 1 of $[7\rceil$; this holds as long as $f \in L^{2}(\Omega)$ and $\Omega$ is a convex polyhedron. However for theorems 3.9 and 3.10 we will need the smoothness of the function $u(x)$ so we will assume $f$ to be smooth. The smoothness condions imposed in this section can be reduced (with a consequence that the estimates we obtain may be weaker) but for simplicity we do not present this here. The order of convergence in $L^{2}(\Omega)$ is $O(\varepsilon)$.

An error estimate like (3.12) for more than two scales is still to be established; since this is not the main focus of this paper, we confine ourselves in this section to the two scale case.

The norms of the FE solutions $u_{1}^{L}$ and $\hat{u}_{1}^{L}$ can be unbounded in $C^{0,1}\left(\Omega, W^{1, \infty}(Y)\right)$ when the mesh size $h_{L} \rightarrow 0$, so an estimate of the form (3.12) in terms of these 
functions is not possible in general. We therefore "postprocess" the FE approximation of $u_{1}$ using the operator $\mathcal{U}^{\varepsilon}$ introduced in [9].

Definition 3.6. For $\Phi \in L^{1}(\Omega \times Y)$ we define

$$
\mathcal{U}^{\varepsilon}(\Phi)(x)=\int_{Y} \Phi\left(\varepsilon\left[\frac{x}{\varepsilon}\right]+\varepsilon z,\left\{\frac{x}{\varepsilon}\right\}\right) d z
$$

where $\lceil x / \varepsilon\rceil$ denotes the "integer" part of $x / \varepsilon$ with respect to $Y$ and $\{x / \varepsilon\}=x / \varepsilon-\lceil x / \varepsilon]$.

We have the following properties of $\mathcal{U}^{\varepsilon}$.

Lemma 3.7. ((97) For $\Phi \in L^{1}(\Omega \times Y)$,

$$
\int_{\Omega} \mathcal{U}^{\varepsilon}(\Phi)(x) d x=\int_{\Omega \times Y} \Phi(x, y) d x d y
$$

Proof Let $I$ be a subset of $\mathbb{Z}^{d}$ such that $\Omega \subset \bigcup_{m \in I} \varepsilon(m+Y)$. The function $\Phi$ is extended by 0 outside $\Omega$. Then

$$
\begin{aligned}
& \int_{\Omega} \int_{Y} \Phi\left(\varepsilon\left[\frac{x}{\varepsilon}\right]+\varepsilon z,\left\{\frac{x}{\varepsilon}\right\}\right) d z d x=\sum_{m \in I} \int_{Y} \int_{m+\varepsilon Y} \Phi\left(\varepsilon m+\varepsilon z,\left\{\frac{x}{\varepsilon}\right\}\right) d x d z \\
& =\varepsilon^{d} \sum_{m \in I} \int_{Y} \int_{Y} \Phi(\varepsilon m+\varepsilon z, y) d z d y=\int_{\Omega \times Y} \Phi(x, y) d x d y .
\end{aligned}
$$

Lemma 3.8. If $\Phi \in C^{1}\left(\bar{\Omega}, C_{\#}(Y)\right)$ then

$$
\int_{\Omega}\left|\Phi\left(x, \frac{x}{\varepsilon}\right)-\mathcal{U}^{\varepsilon}(\Phi)(x)\right|^{2} d x \leq C \varepsilon^{2} .
$$

The constant $C$ depends on $\Phi$.

Proof Since $\Phi \in C^{1}\left(\bar{\Omega}, C_{\#}(Y)\right)$ we have for all $x \in \Omega$ and $z \in Y$

$$
\left|\Phi\left(x, \frac{x}{\varepsilon}\right)-\Phi\left(\varepsilon\left[\frac{x}{\varepsilon}\right]+\varepsilon z, \frac{x}{\varepsilon}\right)\right| \leq C \varepsilon
$$

where $C$ depends on $\Phi$. Then

$$
\int_{\Omega}\left|\int_{Y}\left(\Phi\left(x, \frac{x}{\varepsilon}\right)-\Phi\left(\varepsilon\left[\frac{x}{\varepsilon}\right]+\varepsilon z, \frac{x}{\varepsilon}\right)\right) d y\right|^{2} d x \leq C \varepsilon^{2} .
$$

We can now prove an estimate between the physical solution $u^{\varepsilon}$ and the "contracted" FE solution of the multidimensional limit problem.

Theorem 3.9. Assume that $A(x, y)$ and $f$ are smooth and $\Omega$ is a convex polyhedron. With the full tensor product of the FE spaces in Example 1 with degree $p=1$, we have the asymptotic error estimate

$$
\left\|\nabla_{x} u^{\varepsilon}(x)-\left[\nabla_{x} u^{L}(x)+\mathcal{U}^{\varepsilon}\left(\nabla_{y} u_{1}^{L}\right)(x)\right]\right\|_{L^{2}(\Omega)} \leq c\left(\varepsilon^{1 / 2}+h_{L}\right) .
$$

Proof From Lemma 3.8 and (3.12) we have

$$
\left\|\nabla_{x} u^{\varepsilon}(x)-\nabla_{x} u(x)-\nabla_{y} u_{1}\left(x, \frac{x}{\varepsilon}\right)\right\|_{L^{2}(\Omega)} \leq c \varepsilon^{1 / 2} .
$$

Since $A$ and $f$ are smooth and $\Omega$ is convex, $u(x) \in H^{2}(\Omega)$ and $u_{1}(x, y) \in H^{1}\left(\Omega, H_{\#}^{1}\left(Y_{1}\right)\right)$ (see the next section). From Lemma 3.7 we have then

$$
\left\|\mathcal{U}^{\varepsilon}\left(\nabla_{y} u_{1}\right)(x)-\mathcal{U}^{\varepsilon}\left(\nabla_{y} u_{1}^{L}\right)(x)\right\|_{L^{2}(\Omega)}=\left\|\nabla_{y} u_{1}(x, y)-\nabla u_{1}^{L}(x, y)\right\|_{L^{2}(\Omega \times Y)} \leq c h_{L} .
$$

The result then follows. $Г$ 
One main result of the paper is that for the sparse FE space we have a completely analogous error estimate:

Theorem 3.10. Assume that $A$ and $f$ are smooth and that $\Omega$ is a convex polyhedron. With the sparse tensor product FE space from Example 1 it holds

$$
\left\|\nabla_{x} u^{\varepsilon}(x)-\left[\nabla_{x} u^{L}(x)+\mathcal{U}^{\varepsilon}\left(\nabla_{y} \hat{u}_{1}^{L}\right)(x)\right]\right\|_{L^{2}(\Omega)} \leq c\left(\varepsilon^{1 / 2}+L^{1 / 2} h_{L}\right) .
$$

The proof is analogous to that of Theorem 3.9 if we use that the solution $\left(u,\left\{u_{i}\right\}\right)$ has $H^{2}(\Omega), \hat{\mathcal{H}}_{i}^{1}$-regularity. This will be shown in the next section.

REMARK 7. Theorem 3.10 was stated only for elements of degree $p=1$. Under suitable regularity assumptions for the limiting problem (2.7), we get for $p>1$ the bound

$$
\left\|\nabla_{x} u^{\varepsilon}(x)-\left[\nabla_{x} u^{L}(x)+\mathcal{U}^{\varepsilon}\left(\nabla_{y} \hat{u}_{1}^{L}\right)(x)\right]\right\|_{L^{2}(\Omega)} \leq c\left(\varepsilon^{1 / 2}+L^{1 / 2} h_{L}^{p}\right) .
$$

4. Multiscale Regularity . From Proposition 3.4 and Theorem 3.10 we see that it is possible to approximate the solution of the high dimensional limit problem (2.7) at an optimal convergence rate, provided that the functions $u_{i}$ exhibit joint regularity in each fast variable $y_{k}$, i.e. $u_{i} \in \hat{\mathcal{H}}_{i}^{p}$. In this section we show that this regularity for $\left(u,\left\{u_{i}\right\}\right)$ typically holds under mild regularity conditions on the coefficients $A$.

TheOREM 4.1. Assume that $A\left(x, y_{1}, \ldots, y_{n}\right) \in C^{0,1}\left(\Omega, C_{\#}^{0,1}\left(Y_{1}, \ldots, C_{\#}^{0,1}\left(Y_{n}\right) \ldots\right)\right)$ i.e $A$ is Lipschitz with respect to each $x, y_{1}, \ldots, y_{n}$, and is symmetric. The domain $\Omega$ is supposed to be a convex polyhedron. Then the solution $\left(u,\left\{u_{i}\right\}\right)$ of the problem (2.7) satisfies $u \in H^{2}(\Omega)$ and $u_{i} \in \hat{\mathcal{H}}_{i}^{1}$ for each $i=1, \ldots, n$.

Proof From (2.7) we have that

$$
u_{n}=w_{n l}\left(\frac{\partial u}{\partial x_{l}}+\frac{\partial u_{1}}{\partial y_{1 l}}+\ldots+\frac{\partial u_{n-1}}{\partial y_{(n-1) l}}\right)
$$

(the repeated index $l$ indicates summation from 1 to $d)$ where $w_{n l} \in L^{2}\left(\Omega \times Y_{1} \times \ldots \times\right.$ $\left.Y_{n-1}, H_{\#}^{1}\left(Y_{n}\right)\right)$ is the solution of the variational problem

$$
\int_{\Omega} \int_{Y_{1}} \ldots \int_{Y_{n}} A\left(e_{l}+\nabla_{y_{n}} w_{n l}\right) \cdot \nabla_{y_{n}} \phi_{n} d x d y_{1} \ldots d y_{n}=0
$$

for $\phi_{n} \in \mathcal{D}\left(\Omega \times Y_{1} \times \ldots \times Y_{n}\right), e_{l}$ is the $l^{\text {th }}$ unit vector in $\mathbb{R}^{d}$. Substituting this form of $u_{n}$ back into (2.7) we have

$$
\int_{\Omega} \int_{Y_{1}} \ldots \int_{Y_{n}} A\left(I+\nabla_{y_{n}} w_{n}\right)\left(\nabla_{x} u+\sum_{k=1}^{n-1} \nabla_{y_{k}} u_{k}\right) \cdot \nabla_{y_{n-1}} \phi_{n-1} d x d y_{1} \ldots d y_{n-1}=0
$$

where $w_{n}$ denotes the vector $\left(w_{n 1}, \ldots, w_{n d}\right)$ and $I$ is the unit matrix. Then

$$
u_{n-1}=w_{(n-1) l}\left(\frac{\partial u}{\partial x_{l}}+\frac{\partial u_{1}}{\partial y_{1 l}}+\ldots+\frac{\partial u_{n-2}}{\partial y_{(n-2) l}}\right)
$$

where $w_{(n-1) l} \in L^{2}\left(\Omega \times Y_{1} \times \ldots \times Y_{n-2}, H_{\#}^{1}\left(Y_{n-1}\right)\right)$ is the solution of the variational problem

$$
\int_{\Omega} \int_{Y_{1}} \ldots \int_{Y_{n-1}} A_{n-1}\left(e_{l}+\nabla_{y_{n-1}} w_{(n-1) l}\right) \cdot \nabla_{y_{n-1}} \phi_{n-1} d x d y_{1} \ldots d y_{n-1}=0
$$

where

$$
A_{n-1}=\int_{Y_{n}} A\left(I+\nabla_{y_{n}} w_{n}\right) d y_{n}=\int_{Y_{n}} A\left(I+\nabla_{y_{n}} w_{n}\right) \cdot\left(I+\nabla_{y_{n}} w_{n}\right) d y_{n} .
$$


With $A_{n}=A$, this formula allows to define recursively $A_{i}$ for all $i$ and we have

$$
u_{i}=w_{i l}\left(\frac{\partial u}{\partial x_{l}}+\ldots+\frac{\partial u_{i-1}}{\partial y_{(i-1) l}}\right)
$$

where $w_{i l} \in L^{2}\left(\Omega \times Y_{1} \times \ldots \times Y_{i-1}, H_{\#}^{1}\left(Y_{i}\right)\right)$ is the solution of the problem

$$
\int_{\Omega} \int_{Y_{1}} \ldots \int_{Y_{i}} A_{i}\left(e_{l}+\nabla_{y_{i}} w_{i l}\right) \cdot \nabla_{y_{i}} \phi_{i} d x d y_{1} \ldots d y_{i}=0
$$

for all $\phi_{i} \in \mathcal{D}\left(\Omega \times Y_{1} \times \ldots \times Y_{i}\right)$. The matrix $A_{i}$ is defined as

$$
A_{i}\left(x, y_{1}, \ldots, y_{i}\right)=\int_{Y_{i+1}} A_{i+1}\left(I+\nabla_{y_{i+1}} w_{i+1}\right) \cdot\left(I+\nabla_{y_{i+1}} w_{i+1}\right) d y_{i+1}
$$

for $i<n$. Finally the effective diffusivity is

$$
A_{0}(x)=\int_{Y_{1}} A_{1}\left(I+\nabla_{y_{1}} w_{1}\right) \cdot\left(I+\nabla_{y_{1}} w_{1}\right) d y_{1}
$$

and we have the following "effective" problem for $u$ :

$$
\int_{\Omega} A_{0}(x) \nabla_{x} u \cdot \nabla_{x} \phi=\int_{\Omega} f \phi
$$

for all $\phi \in \mathcal{D}(\Omega)$.

The functions $u_{i}$ are then determined by

$$
u_{i}=w_{i} \cdot\left(I+\nabla_{y_{i-1}} w_{i-1}\right) \ldots\left(I+\nabla_{y_{1}} w_{1}\right) \nabla_{x} u .
$$

Since the matrix $A_{0}$ is Lipschitz with respect to $x$ and the domain $\Omega$ is convex, $u \in H^{2}(\Omega)$. Furthermore since $A_{i}$ are Lipschitz with respect to $x, y_{1}, \ldots, y_{i}, w_{i l} \in$ $C^{0,1}\left(\Omega, C_{\#}^{0,1}\left(Y_{1}, \ldots, H_{\#}^{2}\left(Y_{i}\right) \ldots\right)\right.$ ) (see Gilbarg and Trudinger [13]). From (4.3) we have $u_{i} \in \hat{\mathcal{H}}_{i}^{1}$.

REMARK 8. If the coefficient $A$ is smooth, we solve the cell problems (4.1) as parametric equation for $y_{i}$ in $\mathbb{R}^{d}$ for many points in $\left(x, y_{1}, \ldots, y_{i-1}\right) \in \mathbb{R}^{i d}$. The sparse FE scheme developed in the previous section is applicable to solve these cell problems with reduced complexity.

REMARK 9. If the coeffient $A$ is in $C^{t-1,1}$ with respect to each variable $x, y_{1}, \ldots, y_{n}$ and $f \in H^{t-1}(\Omega)$ then $u \in H^{t+1}(\Omega)$ and $u_{i} \in \hat{\mathcal{H}}_{i}^{t}$ for $i=1, \ldots, n$.

5. Implementation. The sparse tensor product FEM (3.11) leads to solving a linear system

$$
\hat{S} C_{u}^{L}=C_{f}^{L}
$$

where the stiffness matrix $\hat{S}$ has dimension

$$
\hat{N}^{L}:=\sum_{l=0}^{n} d_{l}^{L}
$$

with $d_{0}^{L}=\operatorname{dim} V_{0}^{L}$ and $d_{i}^{L}=\operatorname{dim} \hat{V}_{i}^{L}$.

Due to the large support of the basis functions in the sparse tensor product space, the matrix $\hat{S}$ is non sparse. Storing it requires memory which grows superlinearly in the number of degrees of freedom. In order to build a solution algorithm of log-linear complexity for the high-dimensional limit problem, we avoid forming the matrix explicitly. Rather, we form the matrix out of blocks corresponding to one-scale problems which can be written in terms of tensor products of small matrices. We will show 
that this is possible with log-linear growth of memory in the number of degrees of freedom.

To simplify the exposition, we consider the case where each entry of the matrix $\hat{S}$ is a finite sum of terms of the form $a_{0}(x) a_{1}\left(y_{1}\right) \ldots a_{n}\left(y_{n}\right)$. Specifically, we work with the case where $A$ is isotropic and of the form

$$
A=a_{0}(x) a_{1}\left(y_{1}\right) \ldots a_{n}\left(y_{n}\right) I .
$$

where $I$ is the $d \times d$ identity matrix. Assumption (5.2) is used below in factoring the stiffness matrix $\hat{S}$ to derive a linear complexity matrix vector multiplication. If the matrix $A$ is smooth, but not of product form, it can be approximated by sums of products (5.2) and our matrix vector multiplication algorithm as well as our complexity estimates can still be applied.

REMARK 10 . If $A$ is anisotropic then it is necessary to deal with each single entry of $A$ separately and then adding up the resulting matrices $\hat{S}$. The result for a single entry of $A$ is achieved when the matrix $I$ in (5.2) is not the identity matrix but has only one entry as 1 and others are zero. This can be done by a similar procedure as that that we are going to present below. We note that if $A$ is of the form (5.2) the cell problem (4.1) reduces to a problem in $\mathbb{R}^{d}$ without parameters but if $A$ is more complicated, e.g

$$
A=\left(a_{0}(x) a_{1}\left(y_{1}\right) \ldots a_{n}\left(y_{n}\right)+b_{0}(x) b_{1}\left(y_{1}\right) \ldots b_{n}\left(y_{n}\right)\right) I,
$$

this is no longer the case. To simplify the presentation, we assume in (5.2)

$$
\int_{Y} a_{i}(y) d y=1 \text { for } i=1, \ldots, n .
$$

5.1. Multilevel FE space and preconditioning. It is most convenient to use the wavelet preconditioning procedures to establish bases for $W^{l}, W_{\#}^{l 0}$ and $W_{\#}^{l 1}$. We make the

Assumption 1.

i) For each $j \in \mathbb{N}_{0}{ }^{d}$ there is a set of indices $I^{j} \subset \mathbb{N}_{0}{ }^{d}$ and a family of functions $\psi^{j k} \in L^{2}(\Omega) k \in I^{j}$ such that $\left\{\psi^{j k}:|j|_{\infty} \leq l\right\}$ is a basis for $V^{l}$. There are two constants $0<c_{1}<c_{2}$ such that if $\psi=\sum_{|j|_{\infty} \leq l, k \in I^{j}} \psi^{j k} c_{j k} \in V^{l}$ then

$$
c_{1} \sum_{\substack{|j| \infty<<\\ k \in \Gamma^{j}}}\left|c_{j k}\right|^{2} \leq\|\psi\|_{L^{2}(\Omega)}^{2} \leq c_{2} \sum_{\substack{|j . j|_{\infty}<l \\ k \in I^{j}}}\left|c_{j k}\right|^{2} .
$$

ii) For each $j \in \mathbb{N}_{0}{ }^{d}$ there is a set of indices $I_{0}^{j} \subset \mathbb{N}_{0}{ }^{d}$ and a family of functions $\psi_{0 \#}^{j k} \in H_{\#}^{1}(Y) k \in I_{0}^{j}$ such that $\left\{\psi_{0 \#}^{j k}:|j|_{\infty} \leq l\right\}$ is a basis for the linear space $V_{\#}^{l}$. There are two constants $0<c_{3}<c_{4}$ such that if $\psi=\sum_{|j|_{\infty} \leq l, k \in I_{0}^{j}} \psi_{0 \#}^{j k} c_{j k} \in V_{\#}^{l}$ then

$$
c_{3} \sum_{\substack{|j|_{\infty}<l \\ k \in I_{0}^{j}}}\left|c_{j k}\right|^{2} \leq\|\psi\|_{L^{2}(Y)}^{2} \leq c_{4} \sum_{\substack{|j|_{\infty}<l \\ k \in I_{0}^{j}}}\left|c_{j k}\right|^{2} .
$$

iii) For each $j \in \mathbb{N}_{0}{ }^{d}$ there is a set of indices $I_{1}^{j} \subset \mathbb{N}_{0}{ }^{d}$ and a family of functions $\psi_{1 \#}^{j k} \in H^{1}(Y) / \mathbb{R} k \in I_{1}^{j}$ such that $\left\{\psi_{1 \#}^{j k}:|j|_{\infty} \leq l\right\}$ is a base for the linear space $V_{\#}^{l} / \mathbb{R}$. There are two constants $0<c_{5}<c_{6}$ such that if $\psi=\sum_{|j|_{\infty} \leq l, k \in I^{j}} \psi^{j k} c_{j k} \in V_{\#}^{l} / \mathbb{R}$ then.

$$
c_{5} \sum_{\substack{\mid j j_{\infty}<l \\ k \in I^{j}}}\left|c_{j k}\right|^{2} \leq\|\psi\|_{H^{1}(Y) / \mathbb{R}}^{2} \leq c_{6} \sum_{\substack{|j|_{\infty}<l \\ k \in I_{0}^{j}}}\left|c_{j k}\right|^{2} .
$$

We give some examples of hierarchical wavelet bases in one dimension that we will use later. 
ExAmple 3 . i) Consider the case $\Omega=(0,1)$. The hierarchical base for $L^{2}((0,1))$ can be taken as follows. At level 0 , we choose three piece wise linear function in $(0,1): \psi^{01}$ takes values $(1,0)$ at $(0,1 / 2)$ and 0 in $(1 / 2,1), \psi^{02}$ is the hat function $\phi$ taking values $0,1,0$ at $0,1 / 2,0$ and $\psi^{03}$ takes values $(0,1)$ at $(1 / 2,1)$ and 0 in $(0,1 / 2)$. For other level $j$, we consider the piece wise linear function $\psi$ taking values $(0,-1,2$,$1,0)$ at $(0,1 / 2,1,3 / 2,2)$ and 0 outside $(0,2)$, the left boundary piecewise function $\psi^{\text {left }}$ taking value $(-2,2,-1,0)$ at $(0,1 / 2,1,3 / 2)$ and the right boundary piece wise function $\psi^{\text {right }}$ taking value $(0,-1,2,-2)$ at $(1 / 2,1,3 / 2,2)$. The index set $I^{j}=\left\{1,2, \ldots, 2^{j}\right\}$. The function $\psi^{j 1}(x)=2^{-j / 2} \psi^{\text {left }}\left(2^{j} x\right)$, the functions $\psi^{j k}(x)=2^{-j / 2} \psi\left(2^{j} x-k+3 / 2\right)$ for $k=2, \ldots, 2^{j}-1$ and $\psi^{j 2^{j}}=\psi^{\text {right }}\left(2^{j} x-2^{j}+2\right)$. These functions satisfy Assumption i)

ii) For $Y=(0,1)$, the base can be taken as the one defined in i) above excluding the piecewise linear function $\psi^{01}$ and $\psi^{02}$ and also at other levels we consider the left boundary functions taking values $(0,2,-1,0)$ at $(0,1 / 2,1,3 / 2)$ and the right boundary function taking values $(0,-1,2,0)$ at $(1 / 2,1,3 / 2,2)$ (note that the functions are now defined within an additive constant). We can also consider the piecewise linear hat function $\phi$ in $\mathbb{R}$ taking values $0,1,0$ at $0,1 / 2,0$ and vanishing outside $(0,1)$. The set of indices $I^{j}=\left\{1,2, \ldots, 2^{j}\right\}$. The wavelet functions $\psi^{j k}(x)=2^{-j / 2} \phi\left(2^{j} x-i+1\right)$ satisfies Assumption 1, iii).

From the norm equivalences, it is obvious that the quantity $\left(\sum c_{j k}^{2}\right)^{1 / 2}$ defines an equivalent norm in $L^{2}(\Omega), L^{2}(Y)$ and $H^{1}(Y) / \mathbb{R}$ in $V^{l}, V_{\#}^{l}$ and $V_{\#}^{l} / \mathbb{R}$ respectively. From the coercivity and continuity of the form $B$ and these norm equivalences we have immediately a result on multilevel preconditioning:

PROPOSITION 5.1. The condition number of the stiffness matrix $\hat{S}$ for the sparse grid discretization of the one-scale limit problem has uniformly bounded condition number as $I, \rightarrow \infty$.

With respect to these norms, the increment spaces can be defined as

$$
\begin{aligned}
& W^{l}=\operatorname{Span}\left\{\psi^{j k}:|j|_{\infty}=l\right\}, \\
& W_{\#}^{l 0}=\operatorname{Span}\left\{\psi_{0 \#}^{j k}:|j|_{\infty}=l\right\}, \\
& W_{\#}^{l 1}=\operatorname{Span}\left\{\psi_{1 \#}^{j k}:|j|_{\infty}=l\right\} .
\end{aligned}
$$

For $i \geq 1$, let $\mathbf{j}$ be an $(i+1) \times d$ index matrix of rows $j_{0}, \ldots, j_{i}$ and $\mathbf{k}$ be an $(i+\mathbf{1}) \times d$ index matrix of rows $k_{0}, \ldots, k_{i}$ where $k_{0} \in I^{j_{0}}$ and $k_{\nu} \in I_{0}^{j_{\nu}}$ for $\nu=1, \ldots, i-1$ and $k_{i} \in I_{1}^{j_{i}}$. Denoting by $\psi^{\mathbf{j k}}=\psi^{j_{0} k_{0}} \psi_{0 \#}^{j_{1} k_{1}} \ldots \psi_{1 \#}^{j_{i} k_{i}}$ we can write

$$
\hat{V}_{i}^{L}=\operatorname{Span}\left\{\psi^{\mathbf{j k}}: \sum_{\nu=0}^{i}|j(\nu)|_{\infty} \leq L\right\}
$$

where $j(\nu)$ denotes the $\nu^{\text {th }}$ row of the $(i+1) \times d$ matrix $\mathbf{j}$.

The algebraic excess (or detail space) of $\hat{V}_{i}{ }^{L}$ is then defined as

$$
\hat{W}_{i}^{L}=\operatorname{Span}\left\{\psi^{\mathbf{j k}}: \sum_{\nu=0}^{i}|j(\nu)|_{\infty}=L\right\}=\bigoplus_{\underline{l} \in \mathbb{N}^{i}+1,|\underline{\mid}|=L} W_{\underline{l}}
$$

where

$$
W_{i}^{\underline{l}}=\operatorname{Span}\left\{\psi^{\mathbf{j k}}:|\mathbf{j}(\nu)|=l_{\nu}\right\}
$$

We denote by $\Psi_{i}^{\underline{l}}, \Psi^{l}, \Psi_{\#}^{l 0}, \Psi_{\#}^{l 1}, \Psi$ the column vectors containing the bases of $W_{i}^{\underline{l}}, W^{l}, \stackrel{l 0}{\#}, W_{\#}^{l 1}, V_{0}^{L}$, respectively.

To obtain a log-linear complexity solution algorithm, we make the following assumptions.

ASSUMPTION 2. There exists a number $p>0$ such that 
i) For all $k \in I^{j}$ and $j^{\prime} \in \mathbb{N}^{d}$, the set $\operatorname{supp}\left(\psi^{j k}\right) \cap \operatorname{supp}\left(\psi^{j^{\prime} k^{\prime}}\right)$ has nonempty interior for at most $p^{d} . \Pi_{q=1}^{d} \max \left(1,2^{j_{q}^{\prime}-j_{q}}\right)$ values of $k^{\prime}$.

ii) For all $k \in I^{j}$, the set $\operatorname{supp}\left(\psi^{j k}\right) \cap \operatorname{supp}\left(\psi_{k}^{L}\right)$ where $\left\{\psi_{k^{\prime}}^{L}\right\}$ is the basis of $V_{0}^{L}$ has nonempty interior for at most $p^{d} \sum_{0 \leq\left|j^{\prime}\right| \leq L} \Pi_{q=1}^{d} \max \left(1,2^{j_{q}^{\prime}-j_{q}}\right)$ values of $k^{\prime}$.

iii) For all $k \in I_{0}^{j}$ and $j^{\prime} \in \mathbb{N}^{d}$, the set $\operatorname{supp}\left(\psi_{0 \#}^{j k}\right) \cap \operatorname{supp}\left(\psi_{0 \#}^{j^{\prime} k^{\prime}}\right)$ has nonempty interior for at most $p^{d} . \Pi_{q=1}^{d} \max \left(1,2^{j_{q}^{\prime}-j_{q}}\right)$ values of $k^{\prime}$.

iv) For all $k \in I_{0}^{j}$ and $j^{\prime} \in \mathbb{N}^{d}$, the set $\operatorname{supp}\left(\psi_{0 \#}^{j k}\right) \cap \operatorname{supp}\left(\psi_{1 \#}^{j^{\prime} k^{\prime}}\right)$ has nonempty interior for at most $p^{d} . \Pi_{q=1}^{d} \max \left(1,2^{j_{q}^{\prime}-j_{q}}\right)$ values of $k^{\prime}$.

v) For all $k \in I_{0}^{j}$ and $j^{\prime} \in \mathbb{N}^{d}$, the set $\operatorname{supp}\left(\psi_{1 \#}^{j k}\right) \cap \operatorname{supp}\left(\psi_{1 \#}^{j^{\prime} k^{\prime}}\right)$ has nonempty interior for at most $p^{d} . \Pi_{q=1}^{d} \max \left(1,2^{j_{q}^{\prime}-j_{q}}\right)$ values of $k^{\prime}$.

The assumptions i), iii), iv) and v) hold for the wavelet basis given in the examples above and also for similar wavelet constructions in polygons $[10]$. Assumption ii) holds when for example we assume a wavelet hierarchical basis for $H_{0}^{1}(\Omega)$.

5.2. Matrix-vector multiplication. To achieve linear complexity, the stiffness matrix $\hat{S}$ is never formed explicitly and hence, iterative solution methods are needed for the solution of the linear system (5.1). In our approach, $\hat{S}$ will be generated in factored form and only certain building blocks of the matrix $\hat{S}$ will be computed and stored.

To this end, we define certain block matrices: we denote by $A^{0}$ the stiffness matrix with respect to $a_{0}(x)$ and the basis $\Psi$ of $V_{0}^{L}$; by $A_{l, l^{\prime}}^{i}(i=1, \ldots, n)$ the stiffness matrix describing the interaction between $W_{\#}^{l 1}$ and $W_{\#}^{l^{\prime} 1}$ with respect to $a^{i}(y)$, by $M_{l, l^{\prime}}^{0}$ the mass matrix with respect to $a_{0}(x)$ describing the interaction between $W^{l}$ and $W^{l^{\prime}}$, by $M_{l, l^{\prime}}^{i}(i=1, \ldots, n)$ the mass matrix describing the interaction between $W_{\#}^{l 0}$ and $W_{\#}^{l^{\prime} 0}$ with respect to $a^{i}(y)$, and by $B_{l}^{i \alpha}(i=1, \ldots, n, \alpha=1, \ldots, d)$ the row matrix defined by

$$
\left(B_{l}^{i \alpha}\right)_{1, \nu}=\int_{Y} a_{i}(y) \frac{\partial \Psi_{\#_{\nu}}^{l 1}}{\partial y_{\alpha}} d y
$$

where $\Psi_{\# \nu}^{l 1}$ is the $\nu^{\text {th }}$ function in the base of $W_{\#}^{l 1}$. We further require $C_{l}^{i}(i=$ $1, \ldots, n-1)$, the row matrix defined by

$$
\left(C_{l}^{i}\right)_{1, \nu}=\int_{Y} a_{i}(y) \Psi_{\# \nu}^{l 0} d y
$$

where $\Psi_{\# \nu}^{l 0}$ is the $\nu^{\text {th }}$ function in the base of $W_{\#}^{l 0}$; and $D_{l}^{0 \alpha}(\alpha=1, \ldots, d)$ the matrix defined by

$$
\left(D_{l}^{0 \alpha}\right)_{\mu, \nu}=\int_{\Omega} a_{0}(x) \frac{\partial \Psi_{\mu}}{\partial x_{\alpha}} \Psi_{\nu}^{l 0}(x) d x
$$

where $\Psi_{\mu}$ is the $\mu^{\text {th }}$ function in the base of $V_{0}^{L}$ and $\Psi_{\nu}^{l 0}$ is the $\nu^{\text {th }}$ function in the base of $W^{l 0}$;

$D_{l}^{i \alpha}(i=1, \ldots, n-1, \alpha=1, \ldots, d)$ is the matrix defined by

$$
\left(D_{l, l^{\prime}}^{i \alpha}\right)_{\mu, \nu}=\int_{\Omega} a_{i}(y) \frac{\partial \Psi_{\# \mu}^{l 1}}{\partial y_{\alpha}} \Psi_{\# \nu}^{l^{\prime} 0}(x) d x
$$

where $\Psi_{\# \mu}$ is the $\mu^{\text {th }}$ function in the base of $V_{\#}^{l 1}$ and $\Psi_{\# \nu}^{l^{\prime} 0}$ is the $\nu^{\text {th }}$ function in the base of $W_{\#}^{l^{\prime} 0}$.

From assumption 2 , it it simple to see that

$$
\operatorname{nnz}\left(M_{l, l^{\prime}}^{0}\right) \leq p^{d}\left(\min \left(l, l^{\prime}\right)+1\right)^{d-1} 2^{d \max \left(l, l^{\prime}\right)}
$$


so the memory needed to store all matrices $M_{l, l^{\prime}}^{0}$ for $0 \leq l, l^{\prime} \leq L$ is bounded by

$$
\sum_{l, l^{\prime}=0}^{L}\left(\min \left(l, l^{\prime}\right)+1\right)^{d-1} 2^{d \max \left(l, l^{\prime}\right)} \lesssim(L+1)^{d-1} 2^{d L} .
$$

Similar results are readily seen to hold also for the other matrices, so that the total memory needed to store the matrices $A, B, C, D$ and $M$ grows as $O\left(L^{d-1} 2^{d L}\right)$.

We proceed to derive the matrix vector multiplication algorithm. To this end, we express the bilinear form $B(\cdot, \cdot)$ on $\hat{\mathbf{V}}^{L} \times \hat{\mathbf{V}}^{L}$ in terms of the wavelet basis.

To this end, we write $\hat{u}^{L}=\underline{x}_{u 0} \cdot \Phi, \hat{u}_{i}^{L}=\underline{x}_{u i}^{\underline{l}}, \Phi_{i}^{\underline{l}}, \hat{\phi}_{i}^{L}=\underline{x}_{0} . \Phi, \hat{\phi}_{i}=\underline{x}_{u i}^{\underline{l}} \cdot \Psi_{i}^{\underline{l}}$ where $\underline{l} \in \mathbb{N}_{0}^{i+1}$ and $\underline{l}^{\prime} \in \mathbb{N}_{0}^{i^{\prime}+1}$. Then the bilinear form of the high dimensional limit problem (2.7) has the matrix representation

$$
\begin{aligned}
& B\left(\hat{u}^{L},\left\{\hat{u}_{i}^{L}\right\} ; \hat{\phi},\left\{\hat{\phi}_{i}^{L}\right\}\right)= \\
& \left(A^{0} \underline{x}_{u 0}+\sum_{i=1}^{n} \sum_{0 \leq|\underline{l}| \leq L} \sum_{\alpha=1}^{d} D_{\underline{l}_{0}}^{0 \alpha} \bigotimes_{\nu=1}^{i-1} C_{\underline{l}_{\nu}}^{\nu} \bigotimes B_{\underline{l}_{i}}^{i \alpha} \underline{x}_{u i}^{\underline{\underline{l}}}\right) \cdot \underline{x}_{0}+ \\
& \sum_{i^{\prime}=1}^{n} \sum_{0<\left|\underline{\underline{l}^{\prime}}\right|<L}\left(\sum_{\alpha=1}^{d}\left(D_{\underline{l}^{\prime}(0)}^{0 \alpha}\right)^{\top} \bigotimes_{\nu=1}^{i^{\prime}-1}\left(C_{\underline{l}_{\nu}^{\prime}}^{\nu}\right)^{\top} \bigotimes\left(B_{\underline{l}_{i}^{\prime}}^{i^{\prime} \alpha}\right)^{\top} \underline{x}_{u 0}+\right.
\end{aligned}
$$

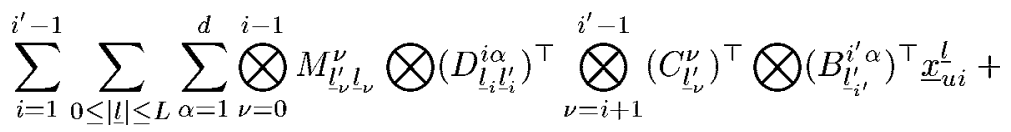

$$
\begin{aligned}
& \sum_{0 \leq|\underline{l}| \leq L} \bigotimes_{\nu=0}^{i^{\prime}-1} M_{\underline{\underline{L}}_{\nu} \underline{L}_{\nu}}^{\nu} \bigotimes A_{{\underline{\underline{l^{\prime}}}}^{\prime} \underline{-}^{\prime}}^{i} \underline{x}_{u i^{\prime}}^{\underline{\underline{l}}}+
\end{aligned}
$$

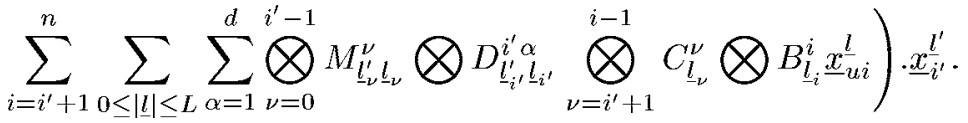

We see that $\hat{S}$ is formed from tensor products of the 'elementary' matrices $A, B, C$, $D$ and $M$ defined above.

The algorithm for applying $\hat{S}$ to a vector with the optimal number of floating point operations is based on [20] and [21].

Step 1: Vector multiplication $D_{\underline{l}_{0}}^{0 \alpha} \bigotimes_{\nu=1}^{i-1} C_{\underline{l}_{\nu}}^{\nu} \otimes B_{\underline{l}_{i}}^{i \alpha} \underline{x}_{u i}^{\underline{l}}$.

Let $R_{i j}$ be the row matrix $C_{\underline{l}_{1}}^{1} \otimes \ldots \otimes B_{\underline{l}_{i}}^{i \alpha}$. The number of nonzero entries of this matrix is bounded by

$$
\left(\underline{l}_{1}+1\right)^{d} \ldots\left(\underline{l}_{i}+1\right)^{d} 2^{d\left(\underline{l}_{1}+\ldots+\underline{l}_{i}\right)} \lesssim(|\underline{\mid l}|+1)^{i d} 2^{d\left(|\underline{\underline{l}}|-\underline{l}_{0}\right)} .
$$

Let $\mathrm{Id}_{\underline{l}_{n} \underline{l}_{0}}$ be the identity matrix whose dimension is $\operatorname{dim} W^{\underline{l}_{0}}$. The multiplication is performed as $D_{\underline{l}_{0}}^{0 \alpha}\left(\operatorname{Id}_{\underline{l}_{0} \underline{l}_{0}} \otimes R_{i j}\right) \underline{x}_{i}^{j}$, which requires a number of floating point operations bounded by $\mathrm{nnz}\left(D_{\underline{l}_{0}}^{0 \alpha}\right)+\mathrm{nnz}\left(\operatorname{Id}_{\underline{l}_{0} \underline{l}_{0}} \otimes R_{i j}\right)$. From Assumption 2, we get

$$
\operatorname{nnz}\left(D_{\underline{l}_{0}}^{0 \alpha}\right) \lesssim \sum_{l=0}^{L}\left(\min \left(l, \underline{l}_{0}\right)+1\right)^{d-1} \cdot 2^{d \max \left(\underline{l}_{0}, l\right)} \lesssim(|\underline{l}|+1)^{d-1} L 2^{d L} .
$$

We also have $\operatorname{dim} W^{\underline{l}_{0}} \sim\left(\underline{l}_{0}+1\right)^{d} 2^{d \underline{l}(0)}$. Thus the floating point operations needed to perform this step over all $i$ and $\underline{l}$ is bounded by

$$
\sum_{i=1}^{n} \sum_{l=0}^{L_{l}} \sum_{|\underline{l}|=l}(l+1)^{(i+1) d} 2^{l d}+(l+1)^{d-1} 2^{L d} L \lesssim \sum_{i=1}^{n} L^{(i+1) d+i} 2^{L d} \lesssim L^{(n+1) d+n} 2^{L d}
$$

(note that the number of vector $\underline{l} \in \mathbb{N}_{0}^{i+1}$ such that $|\underline{l}|=l$ is about $O\left(l^{i}\right)$ ). 
Step 2: Multiplication $\left(D_{\underline{L}_{0}^{\prime}}^{0 \alpha}\right)^{\top} \bigotimes_{\nu=1}^{i^{\prime}-1}\left(C_{\underline{l}_{\nu}^{\prime}}^{\nu}\right)^{\top} \otimes\left(B_{\underline{l}_{i}^{\prime}}^{i^{\prime} \alpha}\right)^{\top} \underline{x}_{0}$.

This multiplication is performed as $\left(\left(R_{i j}\right)^{\top} \otimes \operatorname{Id}_{\underline{l}_{0}^{\prime} \underline{l}_{0}^{\prime}}\right)\left(D_{u l_{0}^{\prime}}^{0 \alpha}\right)^{\top} \underline{x}_{0}$. Similarly as above, the number of floating point operations needed to perform this step over all $i^{\prime}, \underline{l}^{\prime}$ is bounded by $L^{(n+1) d+n} 2^{L d}$.

Step 3: Matrix vector multiplication

$$
\bigotimes_{\nu=0}^{i-1} M_{\underline{l}_{\nu}^{\prime} \underline{l}_{\nu}^{\prime}}^{\nu} \bigotimes\left(D_{\underline{l}_{i} \underline{l}_{i}^{\prime}}^{i \alpha}\right)^{\top} \bigotimes_{\nu=i+1}^{i^{\prime}-1}\left(C_{\underline{l}_{\nu}^{\prime}}^{\nu}\right)^{\top} \bigotimes\left(B_{\underline{l}_{i}^{\prime}}^{i^{\prime} \alpha}\right)^{\top} \cdot \underline{x}_{i}^{\underline{l}}
$$

$\left(1 \leq i \leq i^{\prime}-1\right.$ and $\left.\alpha=1, \ldots, d\right)$. We regard $\underline{l}_{\nu}=0$ for $\nu=i+1, \ldots, i^{\prime}$. From [21] Lemma 5.8, there is a permutation $\sigma$ of $\left(0, \ldots, i^{\prime}\right)$ such that

$$
\forall 0 \leq q \leq i^{\prime}: \quad \sum_{\nu=0}^{q} \underline{l}_{\sigma(\nu)}+\sum_{\nu=q+1}^{i^{\prime}} \underline{l}_{\sigma(\nu)}^{\prime} \leq \max \left(\sum_{\nu=0}^{i} \underline{l}_{\nu}+\sum_{\nu=0}^{i^{\prime}} \underline{l}_{\nu}^{\prime}\right) .
$$

Let $T_{\underline{l}, \underline{l}^{\prime}, q}=\bigotimes_{\nu=1}^{i^{\prime}} U_{\nu}$ where

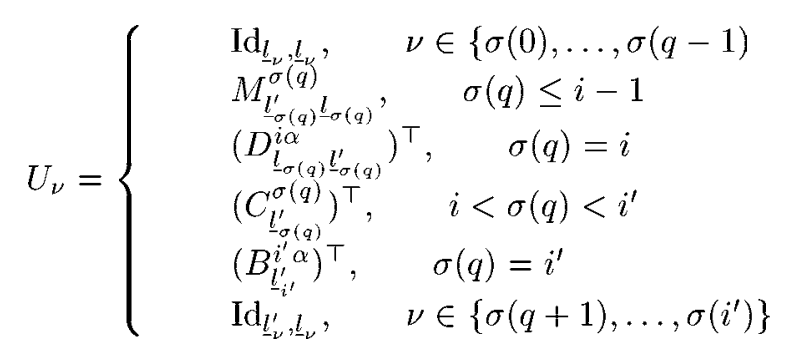

where $\operatorname{Id}_{00}=1, \operatorname{Id}_{\underline{l}_{0}}, \underline{l}_{0}$ is the identity matrix of size $\operatorname{dim} W \underline{\underline{l}}_{0}, \operatorname{Id}_{\underline{L}_{\nu} \underline{\nu}_{\nu}}$ is the identity matrix of size $\operatorname{dim} W_{\#}^{\underline{L_{\nu}}}$ if $0<\nu<i$ and of $\operatorname{size} \operatorname{dim} W_{\#}^{\underline{L}_{1} 1}$ if $\nu=i$; $\operatorname{Id}_{\underline{\underline{L}}_{\nu}^{\prime} \underline{l}_{\nu}^{\prime}}$ is defined similarly (we replace $i$ by $i^{\prime}$ in the definition). Then

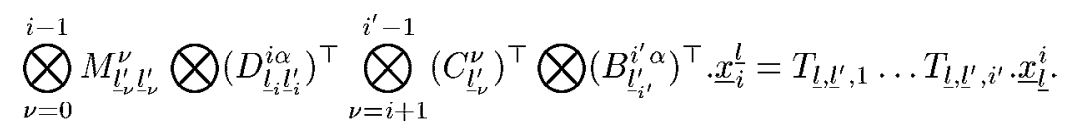

The order of multiplication is essential here to get the following estimate on the number of floating points operations needed. We have

$$
\begin{aligned}
\operatorname{nnz}\left(T_{\underline{l}, \underline{l}^{\prime}, q}\right) & \lesssim \Pi_{\nu=1}^{q-1}\left(\underline{l}_{\nu(q)}+1\right)^{d} \cdot\left(\min \left(\underline{l}_{\sigma(q)}^{\prime}, \underline{l}_{\sigma(q)}\right)+1\right)^{d-1} \cdot \pi_{\nu=q+1}^{i^{\prime}}\left(\underline{l}_{\sigma(\nu)}^{\prime}+1\right)^{d} \\
& \cdot 2^{d\left(\sum_{\nu=1}^{q-1} \underline{l}_{\sigma(\nu)}+\max \left(\underline{l}_{\sigma(q)}^{\prime}, \underline{l}_{\sigma(q)}\right)+\sum_{\sigma=q+1}^{i^{\prime}} \underline{l}_{\sigma(\nu)}\right)} \\
& \lesssim\left(\max \left(|\underline{l}|,\left|\underline{l}^{\prime}\right|\right)+1\right)^{d\left(i^{\prime}+1\right)} 2^{d \max \left(|\underline{l}|, \underline{l}^{\prime} \mid\right)}
\end{aligned}
$$

here we used that $\operatorname{dim} W^{l 0} \simeq(l+1) 2^{d l}$ and $\operatorname{dim} W_{\#}^{l 0}, \operatorname{dim} W_{\#}^{l 1} \simeq(l+1) 2^{d l}$. Thus this matrix vector multiplication needs at most

$$
\sum_{q=1}^{i^{\prime}} \mathrm{nnz}\left(T_{\underline{l}, \underline{l}^{\prime}, q}\right) \lesssim\left(\max \left(|\underline{\mid l}|,\left|\underline{l}^{\prime}\right|\right)+1\right)^{d\left(i^{\prime}+1\right)} 2^{d \max \left(|\underline{l}|,\left|\underline{l}^{\prime}\right|\right)}
$$

floating point operations. The amount of memory needed to store these matrices $\lesssim L^{d(n+1)} 2^{d L}$. The number of floating point operations needed to perform this step over all $l, l^{\prime},|\underline{\mid l}|=l,\left|\underline{l}^{\prime}\right|=l^{\prime}, i$ and $i^{\prime}$ is bounded by

$$
\begin{aligned}
& \sum_{i^{\prime}=1}^{n} \sum_{\substack{l=0 \\
l^{\prime}=0}}^{I} \sum_{\left|\underline{l}^{\prime}\right|=l^{\prime}} \sum_{i<i^{\prime}} \sum_{|\underline{l}|=l}\left(\max \left(|\underline{l}|,\left|\underline{\underline{l}^{\prime}}\right|\right)+1\right)^{d\left(i^{\prime}+1\right)} 2^{d \max \left(|\underline{\underline{l}}|,\left|\underline{l}^{\prime}\right|\right)} \\
\lesssim & \sum_{l, l^{\prime}=1}^{L} l^{n} l^{\prime n}\left(\max \left(l, l^{\prime}\right)+1\right)^{d(n+1)} 2^{d \max \left(l, l^{\prime}\right)} \lesssim L^{2 n+d(n+1)} 2^{d L} .
\end{aligned}
$$


Step 4: We perform the multiplication $\bigotimes_{\nu=0}^{i^{\prime}-1} M_{\underline{\underline{L}}_{\nu}, \underline{l}_{\nu}^{\prime}}^{\nu} \otimes A_{\underline{L}^{\prime}, \underline{l}_{i^{\prime}}}^{i^{\prime}}, \underline{x}_{i^{\prime}}^{\underline{l}}$, similarly. We find an appropriate permutation of $\left(0,1, \ldots, i^{\prime}\right)$ and we write this multiplication in the form $T_{l, l^{\prime}, 1} \ldots T_{l, l^{\prime}, i^{\prime}} \cdot \underline{x}_{i^{\prime}}^{l}$ to get the same estimate as in step 3 for the number of required floating point operations.

Step 5: We perform the multiplication $\bigotimes_{\nu=0}^{i^{\prime}-1} M_{\underline{l}_{\nu}^{\prime}, \underline{L}_{\nu}}^{\nu} \otimes D_{\underline{\underline{L}}^{\prime}, \underline{l}_{i^{\prime}}}^{i^{\prime} \alpha} \otimes_{\nu=i^{\prime}+1}^{i-1} C_{\underline{l}_{\nu}}^{\nu} \otimes B_{\underline{l}_{i}}^{i} x_{i}^{\underline{l}}$, similarly as in step 3. We find a permutation $\sigma$ of $(0,1, \ldots, i)$ for the sets of indices $\left(\underline{l}_{0}, \ldots, \underline{l}_{i}\right)$ and $\left(\underline{l}_{0}^{\prime}, \ldots, \underline{l}_{i^{\prime}}^{\prime}, 0, \ldots, 0\right)\left(i-i^{\prime}\right.$ zeros $)$. We also have the same estimate for the number of floating point operations needed.

The matrix-vector multiplication is summarized in the following algorithm.

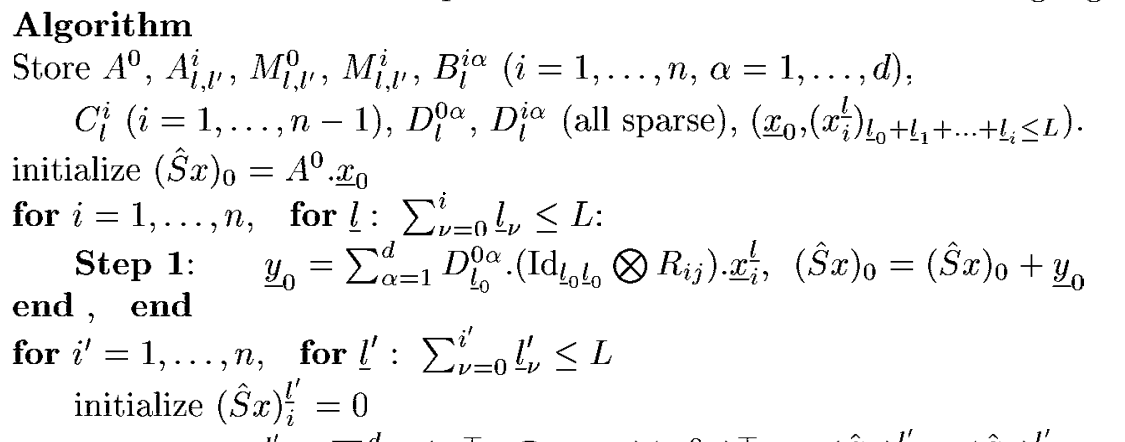

Step 2: $\quad \underline{y}_{i^{\prime}}^{u l^{\prime}}=\sum_{\alpha=0}^{d}\left(R_{i^{\prime} j^{\prime}}^{\top} \otimes \operatorname{Id}_{\underline{l}_{0}^{\prime} \underline{l}_{0}^{\prime}}\right)\left(D_{\underline{l}_{0}^{\prime}}^{0 \alpha}\right)^{\top} \underline{x}_{0}, \quad(\hat{S} x)_{i^{\prime}}^{l^{\prime}}=(\hat{S} x)_{i^{\prime}}^{\underline{l}^{\prime}}+\underline{y}_{i^{\prime}}^{u l^{\prime}}$

Step 3: for $i=1, \ldots, i^{\prime}-1$, for $\underline{l}: \sum_{\nu=0}^{i} \underline{l}_{i} \leq L$

$$
\begin{aligned}
& \underline{y}_{i^{\prime}}^{\underline{l}^{\prime}}=\sum_{N=1}^{d} T_{\underline{l}, \underline{l}^{\prime}, 1} \ldots T_{\underline{l}, \underline{l}^{\prime}, i^{\prime}} \cdot \underline{x}_{\underline{l}}^{i}, \quad(\hat{S} x)_{i^{\prime}}^{\underline{l}^{\prime}}=(\hat{S} x)_{i^{\prime}}^{\underline{l}^{\prime}}+\underline{y}_{i^{\prime}}^{u l^{\prime}} \\
& \text { end, end }
\end{aligned}
$$

Step 4: $\quad \underline{y}_{i^{\prime}}^{l^{\prime}}=\sum_{\alpha=1}^{d} T_{\underline{l}, \underline{l}^{\prime}, 1} \ldots T_{\underline{l}, \underline{l}^{\prime}, i^{\prime}} \cdot \underline{x}_{\underline{l}}^{i}, \quad(\hat{S} x)_{i^{\prime}}^{l^{\prime}}=(\hat{S} x)_{i^{\prime}}^{l^{\prime}}+\underline{y}_{i^{\prime}}^{u l^{\prime}}$

Step 5: for $i=i^{\prime}+1, \ldots, n$, for $\underline{l}: \sum_{\nu=0}^{i} \underline{l}_{i} \leq L$

end, end

$$
\begin{aligned}
& \underline{y}_{i^{\prime}}^{\underline{l}^{\prime}}=\sum_{\alpha=1}^{d} T_{\underline{l}, \underline{l}^{\prime}, 1} \ldots T_{\underline{l}, \underline{l}^{\prime}, i} \cdot \underline{x}_{i}^{\underline{\underline{l}}}, \quad(\hat{S} x)_{\bar{i}^{\prime}}^{\underline{l}^{\prime}}=(\hat{S} x)_{i^{\prime}}^{\underline{l}^{\prime}}+\underline{y}_{i^{\prime}}^{u l^{\prime}} \\
& \text { end, end }
\end{aligned}
$$

We use the conjugate gradient $(\mathrm{cg})$ method to solve the linear problem (5.1). Due to the boundedness of the condition number of the matrix $\hat{S}$, the number of cg-steps required to compute the solution up to a prescribed accuracy is bounded. The number of degrees of freedom equals $\hat{N}_{L}=O\left(2^{d L} L^{n}\right)$. Thus we have shown

TheOREM 5.2. Assuming $u \in H^{2}(\Omega)$ and $u_{i} \in \hat{\mathcal{H}}_{i}^{t}$, the multidimensional limit problem (2.7) is numerically solvable with a relative accuracy of $O\left(\hat{N}_{L}^{-1 / d}\left(\log \hat{N}_{L}\right)^{n(1 / 2+1 / d)}\right)$ in the norm ||$|\cdot|||$ defined in (2.6) at a cost of at most $O\left(\hat{N}_{L}\left(\log \hat{N}_{L}\right)^{n+d(n+1)}\right)$ floating point operations with $O\left(\hat{N}_{L}\left(\log \hat{N}_{L}\right)^{d(n+1)-n}\right)$ memory.

REMARK 11. If $u \in H^{t+1}(\Omega)$ and $u_{i} \in \hat{\mathcal{H}}_{i}^{t}$ the relative accuracy achievable by the sparse FE-discretization with elements of degree $p \geq 1$ is

$$
O\left(\hat{N}_{I}^{-\min (t, p) / d}\left(\log \hat{N}_{L}\right)^{n(1 / 2+1 / d)}\right) .
$$

REMARK 12. So far, we performed the convergence and complexity analysis of the sparse FEM for the numerical solution of the high dimensional limit problem only under the strong regularity hypothesis that $u \in H^{2}(\Omega)$ and that $u_{i} \in \hat{\mathcal{H}}_{i}^{t}$. It is well-known, however, that for problems in polygonal domains the solution $u$ of the limiting problem will not belong to $H^{2}(\omega)$, due to corner singlarities. We emphasize 


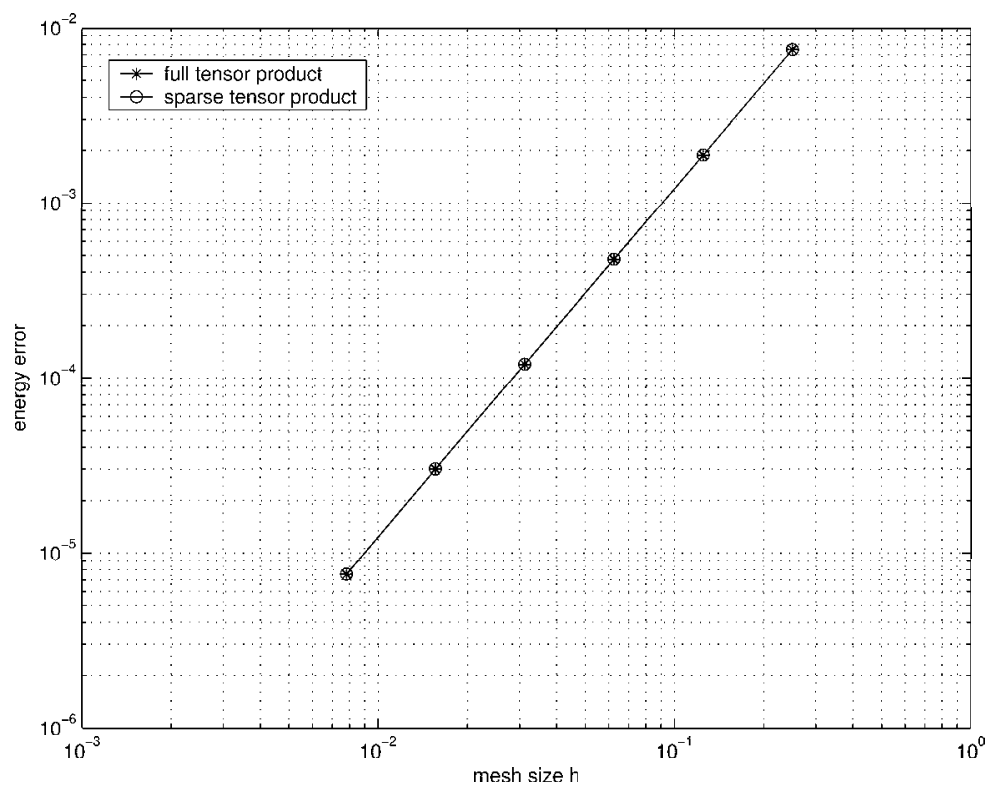

FIG. 6.1. The energy error versus the mesh size $h$

that in such cases our convergence analysis is still valid, if the quasiuniform mesh in the FE space in $\Omega$ is replaced by a mesh with suitable refinement toward the solution's singularities. Since we constructed the members $\hat{V}_{i}^{L}$ of the sparse tensor product space (3.10) from full component FE spaces with hierarchical basis, any type of mesh refinement in these component $\mathrm{FE}$ spaces is admissible in order to resolve solution singularities while preserving our error and complexity estimates; the regularity spaces $\hat{\mathcal{H}}_{i}^{t}$ have to be replaced by weighted spaces, however. See $\lceil 20\rceil$ for sparse grid convergence estimates for component $\mathrm{FE}$ spaces with mesh refinement, in the case $n=1$.

6. Numerical results. In this section, we illustrate the theoretical results presented above. We consider a simple one dimensional two scale problem where $A(x, y)=$ $a_{0}(x) a_{1}(y), a_{0}(x)=1+x$ and $a_{1}(y)=(2 / 3)\left(1+\cos ^{2} 2 \pi y\right), f=-1$ in the domain $\Omega=(0,1)$. The multiscale problem (2.2) becomes

$$
\frac{d}{d x}\left(\frac{2}{3}(1+x)\left(1+\cos ^{2}\left(2 \pi \frac{x}{\varepsilon}\right)\right) \frac{d u^{\varepsilon}}{d x}\right)=1 \quad \text { in } \quad \Omega, \quad u^{\varepsilon}(0)=u^{\varepsilon}(1)=0 .
$$

The two scale limiting equation (2.10) has the exact (homogenized) solution

$$
u(x)=\frac{3}{2 \sqrt{2}}\left(x-\frac{\log (1+x)}{\log 2}\right),
$$

and the scale interaction term

$$
u_{1}(x, y)=\frac{3}{\sqrt{2}}\left(1-\frac{1}{(1+x) \log 2}\right)\left(\frac{1}{2 \pi \sqrt{2}} \tan ^{-1}(\tan 2 \pi y)-y+C\right) .
$$

(note that fixing $x, u_{1}(x, y)$ is determined within an additive constant).

In the sparse FEM, we use the hierarchical base in example 3i) for $L^{2}(\Omega)$ and the hat function base in 3iii) for $H^{1}(Y) / \mathbb{R}$. The number of degrees of freedom for the sparse FEM is $(L+3) 2^{L+1}-2$.

In Figure 6.1, we plot the energy error versus the mesh size $h$ for the full tensor product FE and the sparse tensor product.

We see that the full and sparse tensor product $\mathrm{FE}$ - errors, i.e. $\left\|\left|\left(u-u^{F^{\prime} E^{\prime}}, u_{1}-u_{1}^{f^{\prime} E}\right)\right|\right\| \|^{2}$ and $\left\|\left(u-\hat{u}^{F^{\prime} E^{\prime}}, u_{1}-\hat{u}_{1}^{F^{\prime}}\right)\right\| \|^{2}$, are roughly the same for the same mesh size, which 


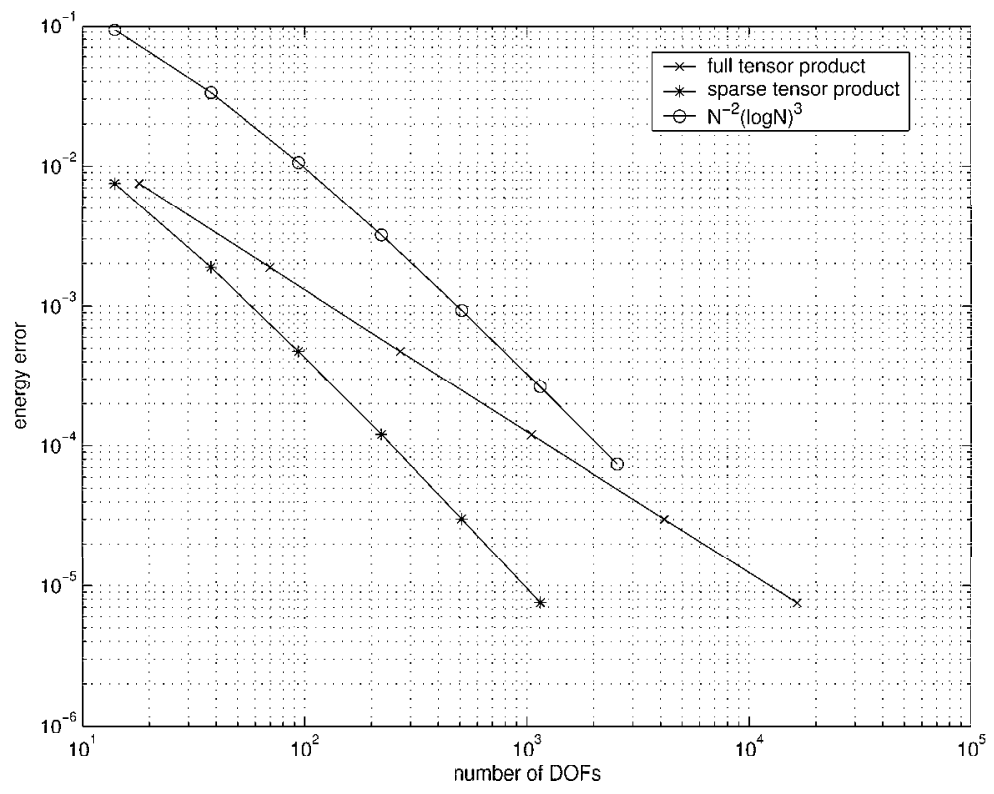

FIG. 6.2. The energy error versus the number of degrees of freedom

illustrates our result that the scale interaction functions $u_{i}\left(x, y_{1}, \ldots, y_{i}\right)$ have sufficient regularity so as to allow a sparse approximation without reducing the convergence rate, in terms of the meshwidth.

In Figure 6.2, we compare the energy error with the number of degrees of freedom $N$. It is clear that the same energy error is obtained with a much smaller number of degrees of freedom in the sparse tensor product FE case as compared to the full tensor product case. We also see that the energy error for the sparse tensor product FEM decreases of order $O\left(N^{-2}(\log N)^{3}\right)$ where $N$ is the number of degrees of freedom.

Next we consider the three scale problem

$$
\frac{d}{d x}\left(\frac{4}{9}(1+x)\left(1+\cos ^{2}\left(2 \pi \frac{x}{\varepsilon_{1}}\right)\right)\left(1+\cos ^{2}\left(2 \pi \frac{x}{\varepsilon_{2}}\right)\right) \frac{d u^{\varepsilon}}{d x}\right)=1 \text { in } \Omega, \quad u^{\varepsilon}(0)=u^{\varepsilon}(1)=0 .
$$

The three scale limiting problem (2.7) has the exact homogenized solution

$$
u(x)=\frac{9}{8}\left(x-\frac{\log (1+x)}{\log 2}\right),
$$

and the scale interaction terms

$$
\begin{gathered}
u_{1}\left(x, y_{1}\right)=\frac{9}{8}\left(1-\frac{1}{(1+x) \log 2}\right)\left(\frac{1}{2 \pi \sqrt{2}} \tan ^{-1}\left(\tan 2 \pi y_{1}\right)+C_{1}\right) \\
u_{2}\left(x, y_{1}, y_{2}\right)=\frac{9}{8}\left(1-\frac{1}{(1+x) \log 2}\right)\left(\frac{1}{2 \pi \sqrt{2}} \tan ^{-1}\left(\tan 2 \pi y_{2}\right)+C_{2}\right) \frac{\sqrt{2}}{1+\cos ^{2} 2 \pi y_{1}} .
\end{gathered}
$$

In Figure 6.3 the energy error is plotted versus the number of degrees of freedom. The predicted convergence behaviour of $O\left(N^{-2}(\log N)^{6}\right)$ does not realize since, in the range of $N$ used for the computation, the logarithmic terms in the convergence estimate still dominate the error. From our convergence analysis, we expect this effect to be even more pronounced for more than three scales.

Acknowledgements. This paper was written when VHH visited the Seminar for Applied Mathematics at the ETH Zurich. He thanks the members of the Institute for their hospitality and for excellent working facilities. 


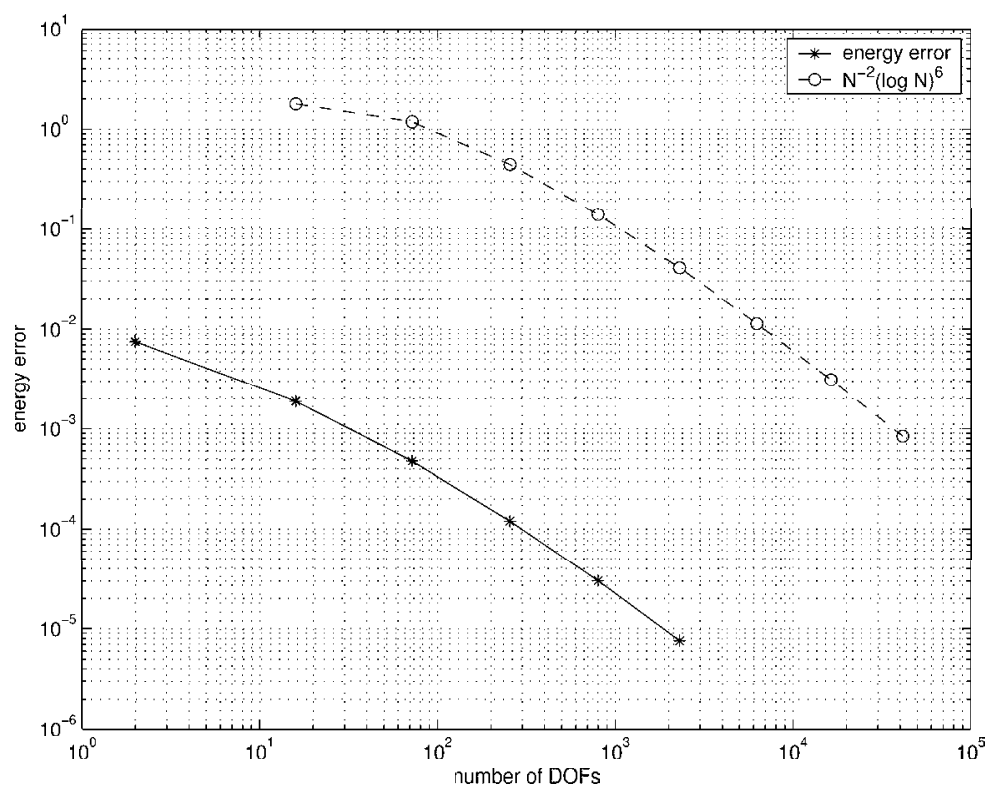

FIG. 6.3. The energy error versus the number of degrees of freedom for three scales

\section{REFERENCES}

[1] G. Allaire, Homogenization and two scale convergence, SIAM J. Math. Anal. 23 (1992), 14821518.

[2] G. Allaire and M. Briane, Multiscale convergence and reiterated homogenization, Proc. Roy. Soc. Edin. 126A (1996), 297-342.

[3] T. Arbogast, Computational aspects of dual porosity models, in Homogenization and Porous Media, U. Hornung ed., p 203-223, New York: Springer 1997

[4] T. Arbogast, J. Douglas and U. Hornung, Derivation of the double porosity model of single phase flow via homogenization theory, SIAM J. Math. Anal. 21 (1990), 823-836.

「5] I. Babuška, Solution of Interface problems by homogenizationI, II, III, SIAM J. Math. Anal.7 (1976), 603-634, 635-645 and 8 (1977), 923-937.

[6] N. Bakhvalov and G. Panasenko, Homogenization: Averaging Process in Periodic Media, Dordrecht:Kluwer, 1989.

[7] A. Bensoussan, J. L. Lions and G. Papanicolaou, Asymptotic Analysis for Periodic Structures, Amsterdam: North Holland, 1978.

[8] P.G. Ciarlet, The Finite Element Method for Elliptic Problems, North Holland Publ. 1978.

[9] D. Cioranescu, A. Damlamian and G. Griso, Periodic unfolding and homogenization, $C . R$. Math. Acad. Sci. Paris 335 (2002), 99-104.

[10] W. Dahmen and R. Stevenson, Element-by-element construction of wavelets satisfying stability and moment conditions, SIAM J. Numer. Ana. 37 (1999), 319-352.

[11] G. Dal Maso, An Introduction to Г-Convergence, Boston: Birkhauser, 1993.

[12] B. Engquist and W. E, The heterogeneous multiscale methods, Commun. Math. Sci. 1 (2003), $87-1.32$.

[13] D. Gilbarg and N. S. Trudinger, Elliptic Partial Differentil Equations of Second Order, Berlin: Springer, 1983.

[14] Z. Chen and T Hou, A mixed multiscale finite element method for elliptic problems with oscillating coefficients Math. Comp 72 (2003), 541-576.

[15] V. Jikov, S. Kozlov and O. Oleinik, Homogenization of differential operators and integral functionals, Berlin: Springer, 1994.

「16] F. Murat, H-convergence, Séminaire d'Analyse Fonctionelle et Numérique de l'Université d'Alger, 1978

[17] A.M. Matache and C. Schwab, Two-scale FEM for Homogenization Problems, R.A.I.R.O. Anal. Numerique 36, (2002) 537-572.

[18] G. Nguetseng, A general convergence result for a functional related to the theorey of homogenization, SIAM J. Math. Anal. 20 (1989), 608-623. 
[19] C. Schwab, High-dimensional finite elements for elliptic problems with multiple scales, Proc. Intern. Congress of Mathematicians, Beijing 2003, pp. 727-734.

$\lceil 20\rceil$ C. Schwab and R.-A Todor, Sparse finite elements for elliptic problems with stochastic data, in press in Numer. Math. (2003).

$\lceil 21\rceil$ C. Schwab and R.-A Todor, Sparse finite elements for stochastic elliptic problems -higher order moments, to appear in Computing (2003).

[22] L. Tartar, Appendix to Sanchez-Palencia, Nonhomogeneous Media and Vibration Theory 127, Berlin, New York: Springer, 1980. 